\title{
A Solution to the Completion Problem for Quasi-Pseudometric Spaces
}

\author{
Athanasios Andrikopoulos \\ Department of Economics, University of Ioannina, P.O. Box 1186, 45110 Ioannina, Greece \\ Correspondence should be addressed to Athanasios Andrikopoulos; aandriko@cc.uoi.gr
}

Received 6 September 2013; Accepted 17 November 2013

Academic Editor: R. Lowen

Copyright (C) 2013 Athanasios Andrikopoulos. This is an open access article distributed under the Creative Commons Attribution License, which permits unrestricted use, distribution, and reproduction in any medium, provided the original work is properly cited.

The different notions of Cauchy sequence and completeness proposed in the literature for quasi-pseudometric spaces do not provide a satisfactory theory of completeness and completion for all quasi-pseudometric spaces. In this paper, we introduce a notion of completeness which is classical in the sense that it is made up of equivalence classes of Cauchy sequences and constructs a completion for any given $T_{0}$ quasi-pseudometric space. This new completion theory extends the existing completion theory for metric spaces and satisfies the requirements posed by Doitchinov for a nice theory of completeness.

\section{Introduction}

A quasi-pseudometric space $(X, d)$ is a set $X$ together with a nonnegative real-valued function $d: X \times X \rightarrow \mathbb{R}$ (called a quasi-pseudometric) such that, for every $x, y, z \in X$, (i) $d(x, x)=0$ and (ii) $d(x, y) \leq d(x, z)+d(z, y)$. If $d$ satisfies the additional condition (iii) $d(x, y)=0$ which implies that $x=y$, then $d$ is called a quasimetric on $X$. A quasimetric is a metric provided that $d(x, y)=d(y, x)$. The conjugate of a quasi-pseudometric $d$ on $X$ is the quasi-pseudometric $d^{-1}$ given by $d^{-1}(x, y)=d(y, x)$. By $d_{s}$ we denote the (pseudo)metric given by $d_{s}=\max \left\{d(x, y), d^{-1}(x, y)\right\}$. Each quasi-pseudometric $d$ on $X$ induces a topology $\tau_{d}$ on $X$ which has as a base the family of $d$-balls $\left\{B_{d}(x, r): x \in X, r>0\right\}$, where $B_{d}(x, r)=\{y \in X: d(x, y)<r\}$. A quasipseudometric space is $T_{0}$ if its associated topology $\tau_{d}$ is $T_{0}$. In that case axiom (i) and the $T_{0}$-condition can be replaced by $\left(\mathrm{i}^{\prime}\right) \forall x, y \in X, d(x, y)=d(y, x)=0 \Leftrightarrow x=y$. It is well known that every metric space has an (up to isometry) unique metric completion. For quasi-pseudometric spaces, the notion of completeness presents serious difficulties. The problem has been approached by several authors in [1-4], and so forth, but the solutions proposed have not solved these difficulties. Since a $T_{0}$ quasi-pseudometric space is a generalization of a metric space (the difference between pseudometrics and metrics is purely topological. In fact a pseudometric $d$ on $X$ is a metric if and only if the topology it generates is $T_{0}$ ), any completion theory for $T_{0}$ quasi-pseudometric spaces should generalize the usual completion theory for metric spaces. Traditionally this is done by generalizing the concept of Cauchy sequence and that of the convergence of a sequence. The appropriate generalization of the notion of Cauchy sequence in quasipseudometric spaces is no longer obvious. There are various generalizations to the notion of Cauchy sequence, but, up to now, none of these generalizations is able to give a satisfying completion theory for all quasi-pseudometric spaces. In fact, there exist many different notions of quasi-pseudometric completeness in the literature. More precisely, this problem has been studied in [3], where seven different notions of Cauchy sequence are presented. (The definitions are that of left and right $p$-Cauchy sequence ( $p$ is the quasimetric) defined by Reilly [5] and Subrahmanyam [6], respectively, p-Cauchy sequence defined by Tan [7], right $K$ - and left $K$ Cauchy sequence defined by Kelly [2], and weakly right $K$ - and weakly left K-Cauchy sequence defined in [3].) By combining the seven notions of Cauchy sequences with the topologies $\tau_{d}, \tau_{d^{-1}}$, and $\tau_{d_{s}}$, we may reach a total of fourteen different definitions of "complete space" (considering the symmetry of using the $d^{-1}$ instead of $d$ ). Since the concept of Cauchy 
sequence generalizes the concept of convergent sequence, it would be desirable that the notion of Cauchy sequence in any quasi-pseudometric space has to be defined in such a manner that $(*)$ every convergent sequence is a Cauchy sequence. However, only one of the definitions presented in [3] satisfies the requirement $(*)$. This definition is the following: a sequence $\left(x_{n}\right)_{n \in \mathbb{N}}$ in a quasi-pseudometric space $(X, d)$ is called Cauchy sequence, if for each $\sigma \in \mathbb{N}$ there are a $y_{\sigma} \in X$ and a $N_{\sigma} \in \mathbb{N}$ such that $d\left(y_{\sigma}, x_{n}\right)<1 / \sigma$ when $n>N_{\sigma}$. Unfortunately, this definition has two serious disadvantages. (i) The property of Cauchyness of a sequence depends not only on its terms but also on some other points which need not to belong to it (see [1, Example 2] and [2, page 88$]$ ). This allows sometimes for a convergent Cauchy sequence to cease Cauchyness if we remove the limit point from the space. (ii) Consider the real line equipped with the quasimetric $d(x, y)=y-x$, if $x \leq y$ and $d(x, y)=1$ if $x>y$. The sequence $(-1 / n)_{n \in \mathbb{N}}$ is a Cauchy one in the sense of the above definition. However (in Doitchinov's words), "in view of the special character of the Sorgenfrey's topology, it seems very inconvenient to regard this sequence as a potentially convergent one."

Doitchinov in [1] introduced a class of quasimetric spaces, for which a satisfactory theory of completeness exists. According to Doitchinov, a notion of Cauchy sequence in any quasimetric space has to be defined in such a manner that this definition provides the properties that convergent sequences are Cauchy, and it agrees with the usual definition for metric spaces. Moreover, the suggested completion must be a monotone operator with respect to inclusion and give rise to the usual pseudometric completion in the pseudometric case. By means of Cauchy sequences which satisfy the above requirements, another concept of Cauchy sequence is proposed and a completion of a quasimetric space is constructed. However, this construction satisfies natural requirements only for balanced quasimetric spaces. (A quasimetric $(X, d)$ is called balanced if $d$ satisfies the following condition: If $\left(x_{n}\right)_{n \in \mathbb{N}}$ and $\left(x_{m}\right)_{m \in \mathbb{N}}$ are two sequence in $(X, d)$ and $x, y \in X$, then from $d\left(x, x_{n}\right) \leq r_{1}$ for each $n, d\left(x_{m}, y\right) \leq r_{2}$ for each $m$, and $\lim _{m, n} d\left(x_{m}, x_{n}\right)=0$ it follows that $d(x, y) \leq r_{1}+r_{2}$.) Unfortunately, Doitchinov's condition of balancedness turns out to be rather restrictive. For instance, balanced $T_{0}$ quasimetrics induce completely regular Hausdorff topologies (see [1, Corollary 3] and [8, Page 208]) and totally bounded balanced $T_{0}$ quasimetrics induce uniformities (see $[9,10])$. Künzi and Kivuvu $[11,12]$ localize Doitchinov's idea of balancedness; that is, they do not work with arbitrary Cauchy filter pairs but only with those that they call balanced Cauchy filter pairs. Before stating the proposed completion theory from Künzi and Kivuvu, we give some definitions from [11]. Let $(X, d)$ be a $T_{0}$-quasimetric space. It is known that $(X, d)$ can be isometrically embedded into a $T_{0}$-quasimetric space $\left(X^{*}, d^{*}\right)$, where the associated metric space $\left(X^{*}, d_{s}^{*}\right)$ is (isometric to) the completion of the metric space $\left(X, d_{s}\right)$. We recall that $(X, d)$ is called bicomplete provided that each $d_{s}$-Cauchy filter converges in $\left(X, d_{s}\right)$. Let $A, B$ be nonempty subsets of $X$. The 2-diameter from $A$ to $B$ is defined by $\Phi_{d}(A, B)=\sup \{d(a, b) \mid a \in A, b \in B\}$. We will say that a pair $(\mathscr{F}, \mathscr{G})$ of filters $\mathscr{F}$ and $\mathscr{G}$ on $X$ is a Cauchy filter pair on $(X, d)$ if $\inf _{F \in \mathscr{F}, G \in \mathscr{G}} \Phi_{d}(F, G)=0$. An arbitrary Cauchy filter pair $(\mathscr{F}, \mathscr{G})$ on $X$ is said to converge to $x \in X$ provided that $\inf _{G \in \mathscr{G}} \Phi_{d}(x, G)=0$ and $\inf _{F \in \mathscr{F}} \Phi_{d}(F, y)=0$. A Cauchy filter pair $(\mathscr{F}, \mathscr{G})$ on $(X, d)$ is said to be balanced on $(X, d)$ if for each $x, y \in X$ we have $d(x, y) \leq \inf _{G \in \mathscr{G}} \Phi_{d}(x, G)+\inf _{F \in \mathscr{F}} \Phi_{d}(F, y)$. The space $(X, d)$ is called $B$-complete provided that each balanced Cauchy filter pair converges in $X$. Let $X^{+}$be the set of all balanced Cauchy filter pairs on $(X, d)$ and let $(\mathscr{F}, \mathscr{G}),\left(\mathscr{F}^{\prime}, \mathscr{G}^{\prime}\right) \in X^{+}$. Then, the formula $d^{+}=\left((\mathscr{F}, \mathscr{G}),\left(\mathscr{F}^{\prime}, \mathscr{G}^{\prime}\right)\right)=\inf _{F \in \mathscr{F}, G^{\prime} \in \mathscr{G}}$ $\Phi_{d}\left(F, G^{\prime}\right)=\inf _{F \in \mathscr{F}, G^{\prime} \in \mathscr{G}} \sup _{f \in F, g^{\prime} \in G^{\prime}} d\left(f, g^{\prime}\right)$ defines the distance from $(\mathscr{F}, \mathscr{G})$ to $\left(\mathscr{F}^{\prime}, \mathscr{G}^{\prime}\right)$. The space $\left(X^{+}, d^{+}\right)$is a quasi-pseudometric space. We define an equivalence relation $\cong$ on the quasi-pseudometric space $\left(X^{+}, d^{+}\right)$as follows: $(\mathscr{F}, \mathscr{G}) \cong\left(\mathscr{F}^{\prime}, \mathscr{G}^{\prime}\right)\left((\mathscr{F}, \mathscr{G}),\left(\mathscr{F}^{\prime}, \mathscr{G}^{\prime}\right) \in\left(X^{+}, d^{+}\right)\right)$if and only if $d^{+}\left((\mathscr{F}, \mathscr{G}),\left(\mathscr{F}^{\prime}, \mathscr{G}^{\prime}\right)\right)=0$ and $d^{+}\left(\left(\mathscr{F}^{\prime}, \mathscr{G}^{\prime}\right),(\mathscr{F}, \mathscr{G})\right)=0$. Let $\widehat{X^{+}}$be the set of all equivalence classes $q_{\widehat{x^{+}}}(\mathscr{F})$, where $\mathscr{F} \in X^{+}$, with respect to $\cong$. Then, $\widehat{d^{+}}$on $\frac{X^{+}}{X^{+}}$defined by $\widehat{d^{+}}\left(q_{\widehat{X^{+}}}(\mathscr{F}), q_{\widehat{X^{+}}}(\mathscr{G})\right)=d^{+}(\mathscr{F}, \mathscr{G})$ whenever $\mathscr{F}, \mathscr{G} \in X^{+}$ determines a $T_{0}$-quasimetric $\widehat{d^{+}}$on $\widehat{X^{+}}$. According to [11, Lemma 5] we can identify $\left(\widehat{X^{+}}, \widehat{d^{+}}\right)$with the subspace $\left(X^{\beta}, d^{\beta}\right)$ of all balanced Cauchy filter pairs on $(X, d)$ that are minimal elements in the space $\left(X^{+}, d^{+}\right)$of all balanced Cauchy filter pairs on $(X, d)$. Künzi and Kivuvu in [11, Theorem 2] prove that if $(X, d)$ is a quasi-pseudometric space then $\left(X^{\beta}, d^{\beta}\right)$ is $B$-complete. The space $\left(X^{\beta}, d^{\beta}\right)$ will be called the standard $B$-completion of $(X, d)$. The authors also prove that the constructed standard $B$-completion extends the bicompletion of every $T_{0}$-quasimetric space. In contrast to $D$-completion, the $B$-completion can be strictly larger than the bicompletion in totally bounded $T_{0}$-quasimetric spaces (see [12, Example 8]). But, sometimes, the B-completion coincides with the bicompletion (see [11, Example 4]). In the present paper, we develop a nonsymmetric completion theory which gives a solution to the problem and extends naturally the existing completion theory for metric spaces. The following example shows that the proposed completion differs from the $B$-completion.

Example 1 (see [2, Example 5.4]). Let $X=\{-1 /(n+1), 1 /(n+$ $1), n \in \mathbb{N}\}$. For each $x, y \in X$, let

$$
d(x, y)= \begin{cases}1 & \text { if } x<0<y \\ 0 & \text { if } y \leq x \\ \min \{1,|x-y|\} & \text { otherwise. }\end{cases}
$$

It is easy to check that $(X, d)$ is a $T_{0}$-quasi-pseudometric space. Let $(\mathscr{F}, \mathscr{G})$ be the filter pair on $X$ generated by $\langle(1 /(n+$ $\left.1))_{n \in \mathbb{N}},(-1 /(n+1))_{n \in \mathbb{N}}\right\rangle$. We have that $(\mathscr{F}, \mathscr{G})$ is a Cauchy filter pair on $(X, d)$, which is not balanced, since

$$
\begin{aligned}
1= & d\left(\frac{-1}{4}, \frac{1}{4}\right) \not \inf _{G \in \mathscr{G}} \Phi_{d}\left(\frac{-1}{4}, G\right) \\
& +\inf _{F \in \mathscr{F}} \Phi_{d}\left(F, \frac{1}{4}\right)=\frac{1}{4}+\frac{1}{4}=\frac{1}{2} .
\end{aligned}
$$

Therefore, the $B$-completion $\left(X^{\beta}, d^{\beta}\right)$ of $(X, d)$ does not add new points to $(X, d)$. In the proposed completion theory, 
the pair $\left\langle(1 /(n+1))_{n \in \mathbb{N}},(-1 /(n+1))_{n \in \mathbb{N}}\right\rangle$ is a $\kappa$-cut in $(X, d)$ (see Definition 8$)$. This $\kappa$-cut defines a new point $\widetilde{x_{0}}$ in a complete quasi-pseudometric space $(\widetilde{X}, \widetilde{d})$ (see Theorem 31 ). If the mapping $\widetilde{\phi}$ is the isometric embedding (a map $f: X \rightarrow$ $Y$ between quasi-pseudometric spaces is called an isometric embedding if $d(f(x), f(y))=d(x, y)$ for all $x, y \in X$ and it is called an isometry if it is a surjective isometric embedding) $\widetilde{\phi}:(X, d) \rightarrow(\widetilde{X}, \widetilde{d})$ such that $\widetilde{\phi}(X)$ is a dense subspace of $(\widetilde{X}, \widetilde{d})$, then $\left(\widetilde{\phi}(-(1 /(n+1)))_{n \in \mathbb{N}}\right.$ and $(\widetilde{\phi}(1 /(n+1)))_{n \in \mathbb{N}}$ converge to $\widetilde{x_{0}}$ with respect to $\tau_{\tilde{d}}$ and $\tau_{\widetilde{d}^{-1}}$, respectively.

In this paper, (i) we propose a solution to the problem of quasi-pseudometric completion, called $\kappa$-completion; and (ii) we show that the proposed definition of Cauchy sequence and the constructed completion satisfy the requirements posed by Doitchinov for a satisfactory theory of completion of quasi-pseudometric spaces. To do that, we introduce the notion of $\kappa$-cut which, in a sense, is a generalization of the notion of the equivalence class of Cauchy sequences in metric spaces. This notion is inspired by the notion of the MacNeilleDedekind's cut [13] (see also [14]). In order to construct a $\kappa$ cut, we make use of pairs "sequence-cosequence" as in the Doitchinov's quasimetric completion. The notion of $\kappa$-cut (a) allows us to restrict the "area" where a new point must stand and (b) enables us to construct the $\kappa$-completion by using Cauchy sequences instead of $\kappa$-cuts.

\section{2. $\kappa$-Cauchy Cut}

Throughout the paper $(X, d)$ will be an arbitrary quasipseudometric space, except the cases when it is explicitly stated that the space is $T_{0}$. According to Doitchinov a sequence $\left(y_{\sigma}\right)_{\sigma \in \mathbb{N}}$ is called a cosequence to $\left(x_{n}\right)_{n \in \mathbb{N}}$, if for any $\varepsilon>0$ there are $\sigma_{\varepsilon}, n_{\varepsilon} \in \mathbb{N}$ such that $d\left(y_{\sigma}, x_{n}\right)<\varepsilon$ when $\sigma \geq \sigma_{\varepsilon}$ and $n \geq n_{\varepsilon}$. In this case, we write $d\left(y_{\sigma}, x_{n}\right) \rightarrow 0$ or $\lim _{\sigma, n} d\left(y_{\sigma}, x_{n}\right)=0$. Generally, given two sequences $\left(x_{n}\right)_{n \in \mathbb{N}}$ and $\left(x_{m}\right)_{m \in \mathbb{N}}$ in $(X, d)$, we write $\lim _{n, m} d\left(x_{n}, x_{m}\right)=r$ if for any $\varepsilon>0$ there are $n_{\varepsilon}, m_{\varepsilon} \in \mathbb{N}$ such that $\left|d\left(x_{n}, x_{m}\right)-r\right|<\varepsilon$ when $n \geq n_{\varepsilon}$ and $m \geq m_{\varepsilon}$.

Definition 2 (see [3]). A sequence $\left(x_{n}\right)_{n \in \mathbb{N}}$ of a quasipseudometric space $(X, d)$ is called right (resp. left) $K$ Cauchy, if for any $\varepsilon>0$ there is an $n_{\varepsilon} \in \mathbb{N}$ such that $d\left(x_{n}, x_{n^{\prime}}\right)<\varepsilon$ for each $n \geq n^{\prime} \geq n_{\varepsilon}$ (resp. $n^{\prime} \geq n \geq n_{\varepsilon}$ ). One calls $n_{\varepsilon}$ (resp. $x_{n_{\varepsilon}}$ ) the extreme index (resp. extreme point) of $\left(x_{n}\right)_{n \in \mathbb{N}}$ for $\varepsilon$.

Definition 3. Let $(X, d)$ be a quasi-pseudometric space and let $\left(x_{n}\right)_{n \in \mathbb{N}},\left(x_{m}\right)_{m \in \mathbb{N}}$ be two sequences on it. One says that $\left(x_{n}\right)_{n \in \mathbb{N}}$ is right (resp. left) $d$-cofinal to $\left(x_{m}\right)_{m \in \mathbb{N}}$, if for each $\varepsilon>0$ there exists $n_{\varepsilon} \in \mathbb{N}$ satisfying the following property: for each $n \geq n_{\varepsilon}$ there exists $m_{n} \in \mathbb{N}$ such that $d\left(x_{m}, x_{n}\right)<\varepsilon$ (resp. $\left.d\left(x_{n}, x_{m}\right)<\varepsilon\right)$ whenever $m \geq m_{n}$. The sequences $\left(x_{n}\right)_{n \in \mathbb{N}}$ and $\left(x_{m}\right)_{m \in \mathbb{N}}$ are right (resp. left) $d$-cofinal if $\left(x_{n}\right)_{n \in \mathbb{N}}$ is right (resp. left) $d$-cofinal to $\left(x_{m}\right)_{m \in \mathbb{N}}$ and vice versa.

Proposition 4. Let $\left(x_{n}\right)_{n \in \mathbb{N}}$ be a right $K$-Cauchy (left $K$ Cauchy) sequence in a quasi-pseudometric space $(X, d)$ with a subsequence $\left(x_{n_{\zeta}}\right)_{n_{\zeta} \in \mathbb{N}}$. Then, $\left(x_{n}\right)_{n \in \mathbb{N}}$ and $\left(x_{n_{\zeta}}\right)_{n_{\zeta} \in \mathbb{N}}$ are right (resp. left) $d$-cofinal.

Proof. Let $\left(x_{n}\right)_{n \in \mathbb{N}}$ and $\left(x_{n_{\zeta}}\right)_{n_{\zeta} \in \mathbb{N}}$ be as in the statement of the proposition. Let $\varepsilon>0$ be given. We know that from some index $n_{\varepsilon}$ onwards we have that $n \geq n^{\prime} \geq n_{\varepsilon}$ implies that $d\left(x_{n}, x_{n^{\prime}}\right)<\varepsilon / 2$. Now if $n \geq n_{\varepsilon}$ we can find $N_{\zeta} \in \mathbb{N}$ such that $N_{\zeta}>n$ (as the $n_{\zeta}$ form a strictly increasing sequence of integers). Hence, for each $n_{\zeta} \geq N_{\zeta}$ we have $d\left(x_{n_{\zeta}}, x_{n}\right)<$ $d\left(x_{n_{\zeta}}, x_{N_{\zeta}}\right)+d\left(x_{N_{\zeta}}, x_{n}\right)<\varepsilon / 2+\varepsilon / 2=\varepsilon$. Hence, $\left(x_{n}\right)_{n \in \mathbb{N}}$ is right $d$-cofinal to $\left(x_{n_{\zeta}}\right)_{n_{\zeta} \in \mathbb{N}}$. The converse is similar.

Proposition 5. In every quasi-pseudometric space $(X, d)$, two right $d$-cofinal right $K$-Cauchy sequences have the same cosequences.

Proof. Let $\left(x_{n}\right)_{n \in \mathbb{N}}$ and $\left(x_{m}\right)_{m \in \mathbb{N}}$ be two right $d$-cofinal sequences. Suppose that $\left(y_{\sigma}\right)_{\sigma \in \mathbb{N}}$ is a cosequence of $\left(x_{m}\right)_{m \in \mathbb{N}}$. Fix an $\varepsilon>0$. Then there exists $\sigma_{\varepsilon}, m_{\varepsilon} \in \mathbb{N}$ such that $d\left(y_{\sigma}, x_{m}\right)<\varepsilon / 2$ for $\sigma \geq \sigma_{\varepsilon}$ and $m \geq m_{\varepsilon}$. On the other hand, there is $n_{\varepsilon} \in \mathbb{N}$ with the following property: for each $n \geq n_{\varepsilon}$ there exists $m_{n} \in \mathbb{N}$ such that $d\left(x_{m}, x_{n}\right)<\varepsilon / 2$ whenever $m \geq m_{n}$. If $m(n)=\max \left\{m_{\varepsilon}, m_{n}\right\}$, then $d\left(y_{\sigma}, x_{n}\right) \leq$ $d\left(y_{\sigma}, x_{m(n)}\right)+d\left(x_{m(n)}, x_{n}\right)<\varepsilon$ for each $\sigma \geq \sigma_{\varepsilon}, n \geq n_{\varepsilon}$. Hence, $\left(y_{\sigma}\right)_{\sigma \in \mathbb{N}}$ is a cosequence of $\left(x_{n}\right)_{n \in \mathbb{N}}$.

Similarly we can prove the following proposition.

Proposition 6. In every quasi-pseudometric space $(X, d)$, two left $d$-cofinal left K-Cauchy sequences are cosequence of the same sequences. lary.

The two previous propositions imply the following corol-

Corollary 7. In every quasi-pseudometric space $(X, d)$, two right (left) $d$-cofinal sequences have the same limit points for $d\left(\right.$ resp. $\left.d^{-1}\right)$.

In the following definition, without loss of generality, we may suppose that, for $\varepsilon^{\prime} \leq \varepsilon$, it is $n_{\varepsilon^{\prime}} \geq n_{\varepsilon}$.

Definition 8. Let $(X, d)$ be a quasi-pseudometric space. We call $\kappa$-cut in $X$ an ordered pair $\xi=(\mathscr{A}, \mathscr{B})$ of families of right $K$-Cauchy sequences and left $K$-Cauchy sequences, respectively, with the following properties.

(i) For any $\left(x_{n}\right)_{n \in \mathbb{N}} \in \mathscr{A}$ and any $\left(y_{\sigma}\right)_{\sigma \in \mathbb{N}} \in \mathscr{B}$ there holds

$$
\lim _{\sigma, n} d\left(y_{\sigma}, x_{n}\right)=0
$$

(ii) Any two members of the family $\mathscr{A}$ (resp. $\mathscr{B}$ ) are right (resp. left) $d$-cofinal.

(iii) The classes $\mathscr{A}$ and $\mathscr{B}$ are maximal with respect to set inclusion.

We call $\mathscr{A}$ (resp. $\mathscr{B})$ first (resp. second) class of $\xi$. In what follows, for simplicity of the proofs, we call the elements of $\mathscr{B}$ left $K$-Cauchy consequences of the elements of $\mathscr{A}$. 
Definition 9. To every $x \in X$ one chooses a $\kappa$-cut $\phi(x)=$ $(\mathscr{A}, \mathscr{B})$, where $\mathscr{A}$ consists of right $K$-Cauchy sequences which converges to $x$ with respect to $\tau_{d}$ and $\mathscr{B}$ consists of left $K$ Cauchy cosequences which converges to $x$ with respect to $\tau_{d^{-1}}$. The sequence $(x)=x, x, x, \ldots$ itself belongs to both of the classes. If there are not right $K$-Cauchy sequences (resp. left $K$-Cauchy cosequences) converging to $x$, then, $\phi(x)=$ $((x), \mathscr{B})$ (resp. $\phi(x)=(\mathscr{A},(x)))$. If $x$ is an isolated point for $\tau_{d}$ and $\tau_{d^{-1}}$, respectively, then $\phi(x)=((x),(x))$.

As we can map $X$ to the set of all $\kappa$-cuts, it is possible that for some $x \in X$ there correspond many $\kappa$-cuts; let $\phi_{i}(x), i \in I_{x}$ (the index set $I_{x}$ depends on the choice of $x$ ). Then, we define

$$
\Phi(x)=\left\{\phi_{i}(x) \mid i \in I_{x}\right\}
$$

Without loss of generality, we assume that $\phi(x)=\phi_{i_{0}}(x)$ for some $i_{0} \in I_{x}$.

Remark 10. If the space $(X, d)$ is $T_{0}$, then the function $\phi$ defined above is an injective function (one-to-one) of $X$ into $\widehat{X}$. Indeed, let $x, y \in X$ be such that $\phi(x)=\phi(y)$. Then, $(x),(y) \in \mathscr{A}_{\phi(x)} \cap \mathscr{B}_{\phi(x)} \cap \mathscr{A}_{\phi(y)} \cap \mathscr{B}_{\phi(y)}$. Thus, $d(x, y)=$ $d(y, x)=0$ which implies that $x=y$.

Definition 11. One calls $\kappa$-Cauchy sequence any right $K$ Cauchy sequence which is member of the first class of a $\kappa$-cut.

Definition 12. Two right $K$-Cauchy sequences $\left(x_{n}\right)_{n \in \mathbb{N}}$ and $\left(x_{m}\right)_{m \in \mathbb{N}}$ defined in a quasi-pseudometric space $(X, d)$ are called $\kappa$-equivalent if every left $K$-Cauchy cosequence to $\left(x_{n}\right)_{n \in \mathbb{N}}$ is a left $K$-Cauchy cosequence to $\left(x_{m}\right)_{m \in \mathbb{N}}$ and vice versa. $X$.

Clearly, $\kappa$-equivalence defines an equivalence relation on

An immediate consequence of Definitions 8, 11, and 12 is the following corollary.

Corollary 13. In every quasi-pseudometric space $(X, d)$, two nonconstant $\kappa$-Cauchy sequences which belong to the same $\kappa$ cut are $\kappa$-equivalent.

Remark 14. In view of Corollary 13, we have that the first class of a $\kappa$-cut is an equivalence class of the $\kappa$-Cauchy sequences that are considered to be equivalent by $\kappa$-equivalence relation.

Definition 15. A quasi-pseudometric space $(X, d)$ is called $\kappa$ complete if every $\kappa$-Cauchy sequence converges.

Definition 16. A $\kappa$-completion of a quasi-pseudometric $(X, d)$ is a $\kappa$-complete quasi-pseudometric space $(\widehat{X}, \widehat{d})$ in which $(X, d)$ can be isometrically embedded as a dense subspace.

If a $\kappa$-cut $\xi=(\mathscr{A}, \mathscr{B})$ does not belong to $\bigcup_{x \in X} \Phi(x)$ we say that $(\mathscr{A}, \mathscr{B})$ is a $\kappa$-gap. The set of all $\kappa$-gaps of $X$ is symbolized by $\bar{X}$. Let us define

$$
\widehat{X}=\phi(X) \bigcup \bar{X}
$$

Throughout the paper, for every $\xi \in \widehat{X}, \mathscr{A}_{\xi}, \mathscr{B}_{\xi}$ denote the two classes of $\xi$. In this case, we write $\xi=\left(\mathscr{A}_{\xi}, \mathscr{B}_{\xi}\right)$.

Definition 17. Let $(X, d)$ be a quasi-pseudometric space. Suppose that $r$ is a nonnegative real number, $\xi^{\prime}, \xi^{\prime \prime} \in \widehat{X}$, $\left(x_{n}^{\prime}\right)_{n \in \mathbb{N}} \in \mathscr{A}_{\xi^{\prime}}$, and $\left(x_{m}^{\prime \prime}\right)_{m \in \mathbb{N}} \in \mathscr{A}_{\xi^{\prime \prime}}$. We put $\widehat{d}\left(\xi^{\prime}, \xi^{\prime \prime}\right) \leq r$ if

(i) $\mathscr{A}_{\xi^{\prime}}=\mathscr{A}_{\xi^{\prime \prime}}$ or

(ii) for each $\varepsilon>0$ there are $n_{\varepsilon}^{\prime}, m_{\varepsilon}^{\prime \prime} \in \mathbb{N}$ such that

$$
d\left(x_{n}^{\prime}, x_{m}^{\prime \prime}\right)<r+\varepsilon
$$

when $n \geq n_{\varepsilon}^{\prime}$ and $m \geq m_{\varepsilon}^{\prime \prime}$. If $\xi^{\prime}=\phi(x)$ for some $x \in X$, then the arbitrary sequence $\left(x_{n}^{\prime}\right)_{n \in \mathbb{N}}$ always coincides with the fixed sequence, for which $x_{n}^{\prime}=x$ for all $n \in \mathbb{N}$. That is, $\widehat{d}\left(\phi(x), \xi^{\prime \prime}\right) \leq$ $r$ if

$$
d\left(x, x_{m}^{\prime \prime}\right)<r+\varepsilon
$$

when $m \geq m_{\varepsilon}^{\prime \prime}$.

Then we let

$$
\widehat{d}\left(\xi^{\prime}, \xi^{\prime \prime}\right)=\inf \left\{r \mid \widehat{d}\left(\xi^{\prime}, \xi^{\prime \prime}\right) \leq r\right\} .
$$

Proposition 18. Let $\xi^{\prime}, \xi^{\prime \prime} \in \widehat{X},\left(x_{n}^{\prime}\right)_{n \in \mathbb{N}} \in \mathscr{A}_{\xi^{\prime}}$ and $\left(x_{m}^{\prime \prime}\right)_{m \in \mathbb{N}} \in$ $\mathscr{A}_{\xi^{\prime \prime}}$. Suppose that $\widehat{d}\left(\xi^{\prime}, \xi^{\prime \prime}\right)=0$. Then, $\left(x_{m}^{\prime \prime}\right)_{m \in \mathbb{N}}$ is right $d$ cofinal to $\left(x_{n}^{\prime}\right)_{n \in \mathbb{N}}$.

Proof. By the suppositions, for each $\varepsilon>0$ there are $n_{\varepsilon}^{\prime}, m_{\varepsilon}^{\prime \prime} \in$ $\mathbb{N}$ such that $d\left(x_{n}^{\prime}, x_{m}^{\prime \prime}\right)<\varepsilon$ whenever $n \geq n_{\varepsilon}^{\prime}$ and $m \geq m_{\varepsilon}^{\prime \prime}$. The rest is evident by Definition 3 .

Proposition 19. The truth of $\widehat{d}\left(\xi^{\prime}, \xi^{\prime \prime}\right) \leq r$ in Definition 17 depends only on $\xi^{\prime}, \xi^{\prime \prime}$, and $r$; it does not depend on the choice of the sequences $\left(x_{n}^{\prime}\right)_{n \in \mathbb{N}}$ and $\left(x_{m}^{\prime \prime}\right)_{m \in \mathbb{N}}$.

Proof. Let $\xi^{\prime}, \xi^{\prime \prime},\left(x_{n}^{\prime}\right)_{n \in \mathbb{N}},\left(x_{m}^{\prime \prime}\right)_{m \in \mathbb{N}}$, and $r$ be as in the supposition of Definition 17. Further, let $\widehat{d}\left(\xi^{\prime}, \xi^{\prime \prime}\right) \leq r$. Then, for each $\varepsilon>0$ there are $n_{\varepsilon}^{\prime}, m_{\varepsilon}^{\prime \prime} \in \mathbb{N}$ such that

$$
d\left(x_{n}^{\prime}, x_{m}^{\prime \prime}\right)<r+\frac{\varepsilon}{3}
$$

when $n \geq n_{\varepsilon}^{\prime}$ and $m \geq m_{\varepsilon}^{\prime \prime}$.

We choose two arbitrary sequences $\left(x_{s}^{\prime}\right)_{s \in \mathbb{N}} \in \mathscr{A}_{\xi^{\prime}}$ and $\left(x_{t}^{\prime \prime}\right)_{t \in \mathbb{N}} \in \mathscr{A}_{\xi^{\prime \prime}}$. Since $\left(x_{n}^{\prime}\right)_{n \in \mathbb{N}}$ is right $d$-cofinal to $\left(x_{s}^{\prime}\right)_{t \in \mathbb{N}}$, there is $N_{\varepsilon}^{\prime} \in \mathbb{N}$ satisfying the following property: for each $n \geq N_{\varepsilon}^{\prime}$ there exists $s_{n} \in \mathbb{N}$ such that

$$
d\left(x_{s}^{\prime}, x_{n}^{\prime}\right)<\frac{\varepsilon}{3}
$$

whenever $s \geq s_{n}$. Let $\widetilde{n}_{\varepsilon}^{\prime}=\max \left\{n_{\varepsilon}^{\prime}, N_{\varepsilon}^{\prime}\right\}$. Then, we have

$$
\begin{aligned}
d\left(x_{s}^{\prime}, x_{m}^{\prime \prime}\right) & \leq d\left(x_{s}^{\prime}, x_{\widetilde{n}_{\varepsilon}^{\prime}}\right)+d\left(x_{\widetilde{n}_{\varepsilon}^{\prime}}, x_{m}^{\prime \prime}\right) \\
& <r+\frac{\varepsilon}{3}+\frac{\varepsilon}{3}=r+\frac{2 \varepsilon}{3}
\end{aligned}
$$

whenever $s \geq s_{\widetilde{n}_{\varepsilon}^{\prime}}$ and $m \geq m_{\varepsilon}^{\prime \prime}$. 
Analogously, since $\left(x_{t}^{\prime \prime}\right)_{t \in \mathbb{N}}$ is right $d$-cofinal to $\left(x_{m}^{\prime \prime}\right)_{m \in \mathbb{N}}$, there is $t_{\varepsilon} \in \mathbb{N}$ satisfying the following property: for each $t \geq$ $t_{\varepsilon}$ there exists $m_{t} \in \mathbb{N}$ such that

$$
d\left(x_{m}^{\prime \prime}, x_{t}^{\prime \prime}\right)<\frac{\varepsilon}{3}
$$

whenever $m \geq m_{t}$. Let $m(t, \varepsilon)=\max \left\{m_{\varepsilon}^{\prime \prime}, m_{t}\right\}$. Then, by (11) we have

$$
\begin{aligned}
d\left(x_{s}^{\prime}, x_{t}^{\prime \prime}\right) & \leq d\left(x_{s}^{\prime}, x_{m(t, \varepsilon)}^{\prime \prime}\right)+d\left(x_{m(t, \varepsilon)}^{\prime \prime}, x_{t}^{\prime \prime}\right) \\
& <r+\frac{2 \varepsilon}{3}+\frac{\varepsilon}{3}=r+\varepsilon
\end{aligned}
$$

when $s \geq s_{\widetilde{n}_{\varepsilon}^{\prime}}$ and $t \geq t_{\varepsilon}$.

Proposition 20. Let $\xi^{\prime}, \xi^{\prime \prime} \in \widehat{X},\left(x_{n}^{\prime}\right)_{n \in \mathbb{N}} \in \mathscr{A}_{\xi^{\prime}}$, and $\left(x_{m}^{\prime \prime}\right)_{m \in \mathbb{N}} \in \mathscr{A}_{\xi^{\prime \prime}}$. Then,

$$
\widehat{d}\left(\xi^{\prime}, \xi^{\prime \prime}\right)=\lim _{n, m} d\left(x_{n}^{\prime}, x_{m}^{\prime \prime}\right)
$$

Proof. Let $\widehat{d}\left(\xi^{\prime}, \xi^{\prime \prime}\right)=r$. Then, for any $\varepsilon>0$ there are $n_{\varepsilon}^{\prime}$, $m_{\varepsilon}^{\prime \prime} \in \mathbb{N}$ such that

$$
d\left(x_{n}^{\prime}, x_{m}^{\prime \prime}\right)<r+\varepsilon
$$

when $n \geq n_{\varepsilon}^{\prime}$ and $m \geq m_{\varepsilon}^{\prime \prime}$. To prove that $r-\varepsilon<d\left(x_{n}^{\prime}, x_{m}^{\prime \prime}\right)$ for $n \geq n_{\varepsilon}^{\prime}$ and $m \geq m_{\varepsilon}^{\prime \prime}$, suppose to the contrary that there exist a subsequence $\left(x_{n_{\zeta}}^{\prime}\right)_{n_{\zeta} \in \mathbb{N}}$ of $\left(x_{n}^{\prime}\right)_{n \in \mathbb{N}}$ and a subsequence $\left(x_{m_{v}}^{\prime \prime}\right)_{m_{v} \in \mathbb{N}}$ of $\left(x_{m}^{\prime \prime}\right)_{m \in \mathbb{N}}$ such that for all $x_{n_{\zeta}}^{\prime}, x_{m_{v}}^{\prime \prime}$ there holds $d\left(x_{n_{\zeta}}^{\prime}, x_{m_{v}}^{\prime \prime}\right) \leq r-\varepsilon$. Then, since Propositions 4,5 , and 6 imply that $\left(x_{n_{\zeta}}^{\prime}\right)_{n_{\zeta} \in \mathbb{N}} \in \mathscr{A}_{\xi^{\prime}}$ and $\left(x_{m_{v}}^{\prime \prime}\right)_{m_{v} \in \mathbb{N}} \in \mathscr{A}_{\xi^{\prime \prime}}$, it follows, according to Definition 17 , that $\widehat{d}\left(\xi^{\prime}, \xi^{\prime \prime}\right) \leq r-\varepsilon$, a contradiction. Therefore, we have $\lim _{n, m} d\left(x_{n}^{\prime}, x_{m}^{\prime \prime}\right)=r$.

Proposition 21. $\widehat{d}$ is a quasi-pseudometric.

Proof. From Definition 17 it follows immediately that $\widehat{d}(\xi, \xi)=0$ and $\widehat{d}\left(\xi, \xi^{\prime}\right) \geq 0$ for all $\xi, \xi^{\prime} \in \widehat{X}$. To prove that $d$ satisfies the triangle inequality let $\xi, \xi^{\prime}, \xi^{\prime \prime} \in \widehat{X}$. We distinguish the following cases.

(i) $\mathscr{A}_{\xi} \neq \mathscr{A}_{\xi^{\prime}}$ and $\mathscr{A}_{\xi^{\prime}} \neq \mathscr{A}_{\xi^{\prime \prime}}$. Suppose that $\widehat{d}\left(\xi, \xi^{\prime}\right)=r_{1}$, $\widehat{d}\left(\xi^{\prime}, \xi^{\prime \prime}\right)=r_{2},\left(x_{n}\right)_{n \in \mathbb{N}} \in \mathscr{A}_{\xi},\left(x_{m}^{\prime}\right)_{m \in \mathbb{N}} \in \mathscr{A}_{\xi^{\prime}}$, and $\left(x_{s}^{\prime \prime}\right)_{s \in \mathbb{N}} \in \mathscr{A}_{\xi^{\prime \prime}}$. Then, for any $\varepsilon>0$ there are $n_{\varepsilon}, m_{\varepsilon}^{\prime}>$ 0 such that $d\left(x_{n}, x_{m}^{\prime}\right)<r_{1}+\varepsilon / 2$ whenever $n \geq n_{\varepsilon}$ and $m \geq m_{\varepsilon}^{\prime}$. Similarly, there are $\widetilde{m}_{\varepsilon}^{\prime}, s_{\varepsilon}^{\prime \prime}>0$ such that $d\left(x_{m}^{\prime}, x_{s}^{\prime \prime}\right)<r_{2}+\varepsilon / 2$ whenever $m \geq \widetilde{m}_{\varepsilon}^{\prime}$ and $s \geq s_{\varepsilon}^{\prime \prime}$. Let $M_{0}=\max \left\{m_{\varepsilon}, \widetilde{m}_{\varepsilon}^{\prime}\right\}$. Then, $d\left(x_{n}, x_{s}^{\prime \prime}\right) \leq d\left(x_{n}, x_{M_{0}}^{\prime}\right)+$ $d\left(x_{M_{0}}^{\prime}, x_{s}^{\prime \prime}\right)<r_{1}+r_{2}+\varepsilon$ for each $n \geq n_{\varepsilon}, s \geq s_{\varepsilon}^{\prime \prime}$. Hence, according to Definition 17, we have

$$
\widehat{d}\left(\xi, \xi^{\prime \prime}\right) \leq r_{1}+r_{2}=\widehat{d}\left(\xi, \xi^{\prime}\right)+\widehat{d}\left(\xi^{\prime}, \xi^{\prime \prime}\right) .
$$

(ii) $\mathscr{A}_{\xi} \neq \mathscr{A}_{\xi^{\prime}}$ and $\mathscr{A}_{\xi^{\prime}}=\mathscr{A}_{\xi^{\prime \prime}}$. Suppose that $\widehat{d}\left(\xi, \xi^{\prime}\right)=r$. Since $\mathscr{A}_{\xi^{\prime}}=\mathscr{A}_{\xi^{\prime \prime}}$, Definition 17 implies that $\widehat{d}\left(\xi, \xi^{\prime}\right) \leq$ $r$ and $\widehat{d}\left(\xi^{\prime}, \xi^{\prime \prime}\right)=0$. Hence

$$
\widehat{d}\left(\xi, \xi^{\prime \prime}\right) \leq r=\widehat{d}\left(\xi, \xi^{\prime}\right)+\widehat{d}\left(\xi^{\prime}, \xi^{\prime \prime}\right) .
$$

(iii) $\mathscr{A}_{\xi}=\mathscr{A}_{\xi^{\prime}}$ and $\mathscr{A}_{\xi^{\prime}} \neq \mathscr{A}_{\xi^{\prime \prime}}$. This case is proved similar to the previous one.

(iv) $\mathscr{A}_{\xi}=\mathscr{A}_{\xi^{\prime}}$ and $\mathscr{A}_{\xi^{\prime}}=\mathscr{A}_{\xi^{\prime \prime}}$. This case is trivial.

After the definition of the quasi-pseudometric $\widehat{d}$, we are going to show that the function $\phi$ of Definition 9 is an isometric embedding of $(X, d)$ into $(\widehat{X}, \widehat{d})$.

Proposition 22. For any $x, y \in X$ there holds $\widehat{d}(\phi(x), \phi(y))=$ $d(x, y)$.

Proof. The assertion of the proposition follows from the fact that $x \in \mathscr{A}_{\phi(x)}, y \in \mathscr{A}_{\phi(y)}$, and Proposition 20.

Proposition 23. For any $\xi \in \widehat{X}$,

(a) if $\left(x_{n}\right)_{n \in \mathbb{N}} \in \mathscr{A}_{\xi}$ then $\widehat{d}\left(\xi, \phi\left(x_{n}\right)\right) \rightarrow 0$;

(b) if $\left(y_{\sigma}\right)_{\sigma \in \mathbb{N}} \in \mathscr{B}_{\xi}$ then $\widehat{d}\left(\phi\left(y_{\sigma}\right), \xi\right) \rightarrow 0$.

Proof. (a) Let $\varepsilon>0$. Then, there exists $n_{\varepsilon}>0$ such that $d\left(x_{n}, x_{n^{\prime}}\right)<\varepsilon / 2$ whenever $n \geq n^{\prime} \geq n_{\varepsilon}$. Now pick a right $K$-Cauchy sequence $\left(x_{m}\right)_{m \in N}$ of $\mathscr{A}_{\xi}$. Since $\left(x_{n}\right)_{n \in \mathbb{N}}$ is right $d$ cofinal to $\left(x_{m}\right)_{m \in \mathbb{N}}$, there is $n_{\varepsilon}^{*} \in \mathbb{N}$ satisfying the following property: for each $n \geq n_{\varepsilon}^{*}$ there exists $m_{n} \in \mathbb{N}$ such that

$$
d\left(x_{m}, x_{n}\right)<\frac{\varepsilon}{2}
$$

whenever $m \geq m_{n}$. Suppose that $\tilde{n}_{\varepsilon}=\max \left\{n_{\varepsilon}, n_{\varepsilon}^{*}\right\}$. Fix an $n \geq \widetilde{n}_{\varepsilon}$ and let $\left(x_{s}\right)_{s \in \mathbb{N}} \in \mathscr{A}_{\phi\left(x_{n}\right)}$. Then, $x_{s} \rightarrow x_{n}$ and so there exists an $s_{\varepsilon} \in \mathbb{N}$ such that $d\left(x_{n}, x_{s}\right)<\varepsilon / 2$ for each $s \geq s_{\varepsilon}$. Hence,

$$
d\left(x_{m}, x_{s}\right) \leq d\left(x_{m}, x_{n}\right)+d\left(x_{n}, x_{s}\right)<\frac{\varepsilon}{2}+\frac{\varepsilon}{2}=\varepsilon
$$

for $m \geq m_{n}$ and $s \geq s_{\varepsilon}$. Since $\left(x_{m}\right)_{m \in \mathbb{N}} \in \mathscr{A}_{\xi}$ and $\left(x_{s}\right)_{s \in \mathbb{N}}$ is an arbitrary right $K$-Cauchy sequence of $\mathscr{A}_{\phi\left(x_{n}\right)}$, we conclude that $\widehat{d}\left(\xi, \phi\left(x_{n}\right)\right)<\varepsilon$. Hence, $\widehat{d}\left(\xi, \phi\left(x_{n}\right)\right)<\varepsilon$ whenever $n \geq \widetilde{n}_{\varepsilon}$.

(b) The proof is similar to that of (a).

Proposition 23 implies the following corollary.

Corollary 24. The set $\phi(X)$ is dense in $(\widehat{X}, \widehat{d})$.

Proposition 25. Let $\left(\xi_{\gamma}\right)_{\gamma \in \mathbb{N}}$ be a nonconstant right $K$-Cauchy sequence of $(\widehat{X}, \widehat{d})$ without last element. Then there exists a right $K$-Cauchy sequence $\left(x_{n}\right)_{n \in \mathbb{N}}$ of $(X, d)$ such that the sequences $\left(\xi_{\gamma}\right)_{\gamma \in \mathbb{N}}$ and $\left(\phi\left(x_{n}\right)\right)_{n \in \mathbb{N}}$ are right $\hat{d}$-cofinal right $K$-Cauchy sequences. 
Proof.

(a) The Construction of the Right K-Cauchy Sequence $\left(x_{n}\right)_{n \in \mathbb{N}}$ of $X$. Let $\left(\xi_{\gamma}\right)_{\gamma \in \mathbb{N}}$ be a $\kappa$-Cauchy sequence in $\widehat{X}$. Without loss of generality we can assume that, for each $\gamma \in \mathbb{N}, \mathscr{A}_{\gamma} \neq \mathscr{A}_{\gamma+1}$. Let $n$ be a nonzero fixed natural number and suppose that $\gamma^{*}(n)$ is the smallest integer $\gamma(n)$ with the property

$$
\widehat{d}\left(\xi_{\gamma}, \xi_{\gamma^{\prime}}\right)<\frac{1}{3 n} \quad \text { for } \gamma \geq \gamma^{\prime} \geq \gamma(n) .
$$

Then, there holds

$$
\gamma^{*}(n) \geq \gamma^{*}\left(n^{\prime}\right) \quad \text { iff } n^{\prime} \geq n .
$$

For each $\gamma \in \mathbb{N}$, we also fix a sequence

$$
\left(x_{n(\gamma)}\right)_{n(\gamma) \in \mathbb{N}} \in \mathscr{A}_{\xi_{\gamma}}
$$

If $\xi_{\gamma}=\phi(x)$ for some $x \in X$, then $x_{n(\gamma)}=x$ for each $n(\gamma) \in \mathbb{N}$. Let

$$
\Lambda(n)= \begin{cases}\gamma^{*}(n)+2 & \text { if } \gamma^{*}(n) \text { is even } \\ \gamma^{*}(n)+3 & \text { if } \gamma^{*}(n) \text { is odd }\end{cases}
$$

Then, for each $\lambda \geq \Lambda(n) / 2$ we have

$$
\widehat{d}\left(\xi_{2 \lambda}, \xi_{2 \lambda-1}\right)<\frac{1}{3 n} .
$$

Hence, since $\mathscr{A}_{2 \lambda-1} \neq \mathscr{A}_{2 \lambda}$, there are $n_{0}(2 \lambda)=n_{0}\left(\gamma^{*}(n), 2 \lambda\right) \epsilon$ $\mathbb{N}$ and $n_{0}(2 \lambda-1) \in \mathbb{N}$ such that

$$
d\left(x_{n(2 \lambda)}, x_{n(2 \lambda-1)}\right)<\frac{1}{3 n}
$$

whenever $n(2 \lambda) \geq n_{0}\left(\gamma^{*}(n), 2 \lambda\right)$ and $n(2 \lambda-1) \geq n_{0}(2 \lambda-1)$. On the other hand, since $\left(x_{n(2 \lambda)}\right)_{n(2 \lambda) \in \mathbb{N}}$ is a right $K$-Cauchy sequence, there exists an $n_{1}(2 \lambda) \in \mathbb{N}$ such that

$$
d\left(x_{n(2 \lambda)}, x_{n^{\prime}(2 \lambda)}\right)<\frac{1}{3 n}
$$

for each $n(2 \lambda) \geq n^{\prime}(2 \lambda) \geq n_{1}(2 \lambda)$.

We put $\tilde{n}\left(\gamma^{*}(n), 2 \lambda\right)=\max \left\{n_{0}\left(\gamma^{*}(n), 2 \lambda\right), n_{1}(2 \lambda)\right\}$. Then, we have

$$
\begin{aligned}
& \tilde{n}\left(\gamma^{*}(n), 2 \lambda\right) \\
& \quad \geq \widetilde{n}\left(\gamma^{*}\left(n^{\prime}\right), 2 \lambda\right) \text { iff } \gamma^{*}(n) \geq \gamma^{*}\left(n^{\prime}\right) .
\end{aligned}
$$

Clearly, (25) and (26) are valid if we replace $n_{0}\left(\gamma^{*}(n), 2 \lambda\right)$ and $n_{1}(2 \lambda)$, respectively, by $\tilde{n}\left(\gamma^{*}(n), 2 \lambda\right)$. Let $\lambda, \lambda^{\prime} \in \mathbb{N}$ be such that $\Lambda(n) / 2 \leq \lambda^{\prime}<\lambda$. Then, since $\gamma^{*}(n) \leq 2 \lambda^{\prime}<2 \lambda-1$, similarly to the above we get

$$
d\left(x_{n(2 \lambda-1)}, x_{n\left(2 \lambda^{\prime}\right)}\right)<\frac{1}{3 n}
$$

whenever $n(2 \lambda-1) \geq n_{1}(2 \lambda-1)$ and $n\left(2 \lambda^{\prime}\right) \geq n_{2}\left(2 \lambda^{\prime}\right)$ for some $n_{1}(2 \lambda-1), n_{2}\left(2 \lambda^{\prime}\right) \in \mathbb{N}$. Let $\tilde{n}(2 \lambda-1)=\max \left\{n_{0}(2 \lambda-\right.$ $\left.1, n_{1}(2 \lambda-1)\right\}$. Then, by putting in $(25), n(2 \lambda)=\tilde{n}\left(\gamma^{*}(n), 2 \lambda\right)$, and $n(2 \lambda-1)=\tilde{n}(2 \lambda-1)$, we obtain that

$$
d\left(x_{\widetilde{n}\left(\gamma^{*}(n), 2 \lambda\right)}, x_{\widetilde{n}(2 \lambda-1)}\right)<\frac{1}{3 n} .
$$

On the other hand (28) implies that

$$
d\left(x_{\tilde{n}(2 \lambda-1)}, x_{n\left(2 \lambda^{\prime}\right)}\right)<\frac{1}{3 n}
$$

for each $n\left(2 \lambda^{\prime}\right) \geq n_{2}\left(2 \lambda^{\prime}\right)$ for some $n_{2}\left(2 \lambda^{\prime}\right) \in \mathbb{N}$. Hence,

$$
d\left(x_{\widetilde{n}(2 \lambda-1)}, x_{n_{2}\left(2 \lambda^{\prime}\right)}\right)<\frac{1}{3 n} .
$$

There are two cases to consider: (i) $\tilde{n}\left(\gamma^{*}(n), 2 \lambda^{\prime}\right) \geq n_{2}\left(2 \lambda^{\prime}\right)$; (ii) $n_{2}\left(2 \lambda^{\prime}\right) \geq \tilde{n}\left(\gamma^{*}(n), 2 \lambda^{\prime}\right)$.

In case (i), (28) implies that

$$
d\left(x_{\widetilde{n}(2 \lambda-1)}, x_{\widetilde{n}\left(\gamma^{*}(n), 2 \lambda^{\prime}\right)}\right)<\frac{1}{3 n} .
$$

Hence,

$$
\begin{aligned}
d\left(x_{\widetilde{n}\left(\gamma^{*}(n), 2 \lambda\right)}, x_{\widetilde{n}\left(\gamma^{*}(n), 2 \lambda^{\prime}\right)}\right)< & d\left(x_{\widetilde{n}\left(\gamma^{*}(n), 2 \lambda\right)}, x_{\widetilde{n}(2 \lambda-1)}\right) \\
& +d\left(x_{\widetilde{n}(2 \lambda-1)}, x_{\widetilde{n}\left(\gamma^{*}(n), 2 \lambda^{\prime}\right)}\right) \\
< & \frac{1}{3 n}+\frac{1}{3 n}<\frac{1}{n} .
\end{aligned}
$$

In case (ii), we have $n_{2}\left(2 \lambda^{\prime}\right) \geq \tilde{n}\left(\gamma^{*}(n), 2 \lambda^{\prime}\right)=$ $\max \left\{n_{0}\left(\gamma^{*}(n), 2 \lambda^{\prime}\right), n_{1}\left(2 \lambda^{\prime}\right)\right\}$; hence (26) implies that

$$
d\left(x_{n_{2}\left(2 \lambda^{\prime}\right)}, x_{\widetilde{n}\left(\gamma^{*}(n), 2 \lambda^{\prime}\right)}\right)<\frac{1}{3 n} .
$$

Therefore, (29), (30), and (34) imply that

$$
\begin{aligned}
d\left(x_{\left.\widetilde{n}\left(\gamma^{*}(n), 2 \lambda\right), x_{\tilde{n}\left(\gamma^{*}(n), 2 \lambda^{\prime}\right)}\right)<}\right. & d\left(x_{\tilde{n}\left(\gamma^{*}(n), 2 \lambda\right)}, x_{\widetilde{n}(2 \lambda-1)}\right) \\
& +d\left(x_{\widetilde{n}(2 \lambda-1)}, x_{n_{2}\left(2 \lambda^{\prime}\right)}\right) \\
& +d\left(x_{n_{2}\left(2 \lambda^{\prime}\right)}, x_{\widetilde{n}\left(\gamma^{*}(n), 2 \lambda^{\prime}\right)}\right) \\
< & \frac{1}{3 n}+\frac{1}{3 n}+\frac{1}{3 n}<\frac{1}{n} .
\end{aligned}
$$

Hence, in any case, we have

$$
d\left(x_{\widetilde{n}\left(\gamma^{*}(n), 2 \lambda\right)}, x_{\widetilde{n}\left(\gamma^{*}(n), 2 \lambda^{\prime}\right)}\right)<\frac{1}{n}
$$

for each $\lambda \geq \lambda^{\prime} \geq \Lambda(n) / 2$.

We consider the set

$$
\Theta=\left\{\tilde{n}\left(\gamma^{*}(n), 2 \lambda\right) \mid n, \lambda \in \mathbb{N}\right\} .
$$

According to (27) we give to $\Theta$ the following order:

$$
\vartheta^{\prime}=\widetilde{n}\left(\gamma^{*}\left(n^{\prime}\right), 2 \lambda^{\prime}\right) \lesssim \vartheta=\widetilde{n}\left(\gamma^{*}(n), 2 \lambda\right)
$$


if and only if

$$
\lambda=\lambda^{\prime}, \quad \gamma^{*}\left(n^{\prime}\right) \leq \gamma^{*}(n), \quad \text { or } \quad \lambda^{\prime}<\lambda
$$

Since $\Theta$ is countable and $\lesssim$ is a linear order, without loss of generality, we may assume that $\Theta \subseteq \mathbb{N}$ and $\lesssim \subseteq \leq$, where $\leq$ is the standard ordering of the natural numbers (this means that $\leq$ is a linear refinement of $\lesssim$ ). We now prove that the sequence

$$
\left(x_{\widetilde{n}\left(\gamma^{*}(n), 2 \lambda\right)}\right)_{\widetilde{n}\left(\gamma^{*}(n), 2 \lambda\right) \in \mathbb{N}}
$$

is a right $K$-Cauchy sequence which satisfies the assumptions of the lemma.

Let $\varepsilon>0$ and $n_{\varepsilon}=\min \{n \mid 2 / n<\varepsilon\}$. Suppose that

$$
\widetilde{n}\left(\gamma^{*}\left(n_{\varepsilon}\right), \Lambda\left(n_{\varepsilon}\right)\right) \leq \widetilde{n}\left(\gamma^{*}\left(n^{\prime}\right), 2 \lambda^{\prime}\right) \leq \widetilde{n}\left(\gamma^{*}(n), 2 \lambda\right) \text {. }
$$

We prove that

$$
d\left(x_{\widetilde{n}\left(\gamma^{*}(n), 2 \lambda\right)}, x_{\widetilde{n}\left(\gamma^{*}\left(n^{\prime}\right), 2 \lambda^{\prime}\right)}\right)<\varepsilon .
$$

If $\lambda^{\prime}=\lambda$ and $\left.\gamma^{*}\left(n^{\prime}\right)=\gamma^{*}(n)\right)$, then $\tilde{n}\left(\gamma^{*}(n), 2 \lambda\right)=$ $\tilde{n}\left(\gamma^{*}\left(n^{\prime}\right), 2 \lambda^{\prime}\right)$. Therefore,

$$
d\left(x_{\widetilde{n}\left(\gamma^{*}(n), 2 \lambda\right)}, x_{\widetilde{n}\left(\gamma^{*}\left(n^{\prime}\right), 2 \lambda^{\prime}\right)}\right)=0<\varepsilon .
$$

Suppose that $\widetilde{n}\left(\gamma^{*}\left(n_{\varepsilon}\right), \Lambda\left(n_{\varepsilon}\right)\right) \leq \widetilde{n}\left(\gamma^{*}\left(n^{\prime}\right), 2 \lambda^{\prime}\right)<\tilde{n}$ $\left(\gamma^{*}(n), 2 \lambda\right)$. Then, we have two cases to consider: (I) $\lambda^{\prime}=\lambda$ and $\gamma^{*}\left(n^{\prime}\right)<\gamma^{*}(n)$; (II) $\lambda^{\prime}<\lambda$. In case (I), we have $\tilde{n}\left(\gamma^{*}\left(n_{\varepsilon}\right), \Lambda\left(n_{\varepsilon}\right)\right) \leq \tilde{n}\left(\gamma^{*}\left(n^{\prime}\right), 2 \lambda\right)<\tilde{n}\left(\gamma^{*}(n), 2 \lambda\right)$. Since $\left(x_{n(2 \lambda)}\right)_{n(2 \lambda) \in \mathbb{N}}$ is a right $K$-Cauchy sequence we have that

$$
\begin{aligned}
d\left(x_{\widetilde{n}\left(\gamma^{*}(n), 2 \lambda\right)}, x_{\widetilde{n}\left(\gamma^{*}\left(n^{\prime}\right), 2 \lambda^{\prime}\right)}\right) & =d\left(x_{\widetilde{n}\left(\gamma^{*}(n), 2 \lambda\right)}, x_{\widetilde{n}\left(\gamma^{*}\left(n^{\prime}\right), 2 \lambda\right)}\right) \\
& <\frac{1}{3 n_{\varepsilon}}<\frac{2}{n_{\varepsilon}}<\varepsilon .
\end{aligned}
$$

We proceed to prove case (II). In this case we have two subcases, $\left(\mathrm{II}_{1}\right) \gamma^{*}\left(n^{\prime}\right)<\gamma^{*}(n)$ and $\left(\mathrm{II}_{2}\right) \gamma^{*}(n) \leq \gamma^{*}\left(n^{\prime}\right)$. In subcase $\left(\mathrm{II}_{1}\right)$ we have $\widetilde{n}\left(\gamma^{*}\left(n_{\varepsilon}\right), \Lambda\left(n_{\varepsilon}\right)\right) \leq \widetilde{n}\left(\gamma^{*}\left(n^{\prime}\right), 2 \lambda^{\prime}\right)<$ $\tilde{n}\left(\gamma^{*}(n), 2 \lambda^{\prime}\right)$. Since $\left(x_{n\left(2 \lambda^{\prime}\right)}\right)_{n\left(2 \lambda^{\prime}\right) \in \mathbb{N}}$ is a right $K$-Cauchy sequence we have that

$$
d\left(x_{\widetilde{n}\left(\gamma^{*}(n), 2 \lambda^{\prime}\right)}, x_{\widetilde{n}\left(\gamma^{*}\left(n^{\prime}\right), 2 \lambda^{\prime}\right)}\right)<\frac{1}{3 n_{\varepsilon}} .
$$

Then, (36) and (45) imply that

$$
\begin{aligned}
d\left(x_{\widetilde{n}\left(\gamma^{*}(n), 2 \lambda\right)}, x_{\widetilde{n}\left(\gamma^{*}\left(n^{\prime}\right), 2 \lambda^{\prime}\right)}\right)< & d\left(x_{\widetilde{n}\left(\gamma^{*}(n), 2 \lambda\right)}, x_{\widetilde{n}\left(\gamma^{*}(n), 2 \lambda^{\prime}\right)}\right) \\
& +d\left(x_{\widetilde{n}\left(\gamma^{*}(n), 2 \lambda^{\prime}\right)}, x_{\widetilde{n}\left(\gamma^{*}\left(n^{\prime}\right), 2 \lambda^{\prime}\right)}\right) \\
< & \frac{1}{n_{\varepsilon}}+\frac{1}{3 n_{\varepsilon}}<\frac{2}{n_{\varepsilon}}<\varepsilon .
\end{aligned}
$$

In order to prove subcase $\left(\mathrm{II}_{2}\right)$, we combine (29) and (30). So, we get

$$
d\left(x_{\widetilde{n}\left(\gamma^{*}(n), 2 \lambda\right)}, \mathrm{x}_{n\left(2 \lambda^{\prime}\right)}\right)<\frac{1}{3 n_{\varepsilon}}+\frac{1}{3 n_{\varepsilon}}=\frac{2}{3 n_{\varepsilon}}
$$

for each $n\left(2 \lambda^{\prime}\right) \geq n_{2}\left(2 \lambda^{\prime}\right)$ for some $n_{2}\left(2 \lambda^{\prime}\right) \in \mathbb{N}$. Now, we have two subcases to consider: the first subcase is that $\tilde{n}\left(\gamma^{*}\left(n^{\prime}\right), 2 \lambda^{\prime}\right) \geq n_{2}\left(2 \lambda^{\prime}\right)$ and the second one is that $n_{2}\left(2 \lambda^{\prime}\right) \geq$ $\tilde{n}\left(\gamma^{*}\left(n^{\prime}\right), 2 \lambda^{\prime}\right) \geq \tilde{n}\left(\gamma^{*}(n), 2 \lambda^{\prime}\right) \geq \tilde{n}\left(\gamma^{*}\left(n_{\varepsilon}\right), \Lambda\left(n_{\varepsilon}\right)\right)$. In the first subcase, (45) implies that

$$
d\left(x_{\widetilde{n}\left(\gamma^{*}(n), 2 \lambda\right)}, x_{\widetilde{n}\left(\gamma^{*}\left(n^{\prime}\right), 2 \lambda\right)}\right)<\frac{2}{3 n_{\varepsilon}}<\frac{2}{n_{\varepsilon}}<\varepsilon .
$$

In the second subcase, we have

$$
d\left(x_{n_{2}\left(2 \lambda^{\prime}\right)}, x_{\widetilde{n}\left(\gamma^{*}\left(n^{\prime}\right), 2 \lambda^{\prime}\right)}\right)<\frac{1}{3 n_{\varepsilon}} .
$$

Therefore, (45) and (49) imply that

$$
\begin{aligned}
d\left(x_{\widetilde{n}\left(\gamma^{*}(n), 2 \lambda\right)}, x_{\widetilde{n}\left(\gamma^{*}\left(n^{\prime}\right), 2 \lambda^{\prime}\right)}\right)< & d\left(x_{\widetilde{n}\left(\gamma^{*}(n), 2 \lambda\right)}, x_{n_{2}\left(2 \lambda^{\prime}\right)}\right) \\
& +d\left(x_{n_{2}\left(2 \lambda^{\prime}\right)}, x_{\widetilde{n}\left(\gamma^{*}\left(n^{\prime}\right), 2 \lambda^{\prime}\right)}\right) \\
< & \frac{2}{3 n_{\varepsilon}}+\frac{1}{3 n_{\varepsilon}}<\frac{2}{n_{\varepsilon}}<\varepsilon .
\end{aligned}
$$

Hence, in any case the inequality (42) is invalid.

(b) $\left(\phi\left(x_{\tilde{n}\left(\gamma^{*}(n), 2 \lambda\right)}\right)\right)_{\tilde{n}\left(\gamma^{*}(n), 2 \lambda\right) \in \mathbb{N}}$ and $\left(\xi_{\gamma}\right)_{\gamma \in \mathbb{N}}$ Are Right $\widehat{d}$ Cofinal. We first show that $\left(\xi_{\gamma}\right)_{\gamma \in \mathbb{N}}$ is right $\widehat{d}$-cofinal to $\left(\phi\left(x_{\tilde{n}\left(\gamma^{*}(n), 2 \lambda\right)}\right)\right)_{\tilde{n}\left(\gamma^{*}(n), 2 \lambda\right) \in \mathbb{N}}$. Fix an $\varepsilon>0$ and let $N_{\varepsilon}=$ $\min \{n \mid 1 / 3 n<\varepsilon\}$. Suppose that $\gamma \geq \Lambda\left(N_{\varepsilon}\right)$. Then, we define $\left(\widetilde{n}\left(\gamma^{*}(n), 2 \lambda\right)\right)_{\gamma}=\left(\widetilde{n}\left(\gamma^{*}\left(N_{\varepsilon}\right), 2 \lambda_{\gamma}\right)\right.$, where $2 \lambda_{\gamma}=\gamma+$ $\bmod (\gamma, 2)+2$.

We prove that

$$
d\left(\phi\left(x_{\widetilde{n}\left(\gamma^{*}(n), 2 \lambda\right)}\right), \xi_{\gamma}\right)<\varepsilon
$$

for each $\tilde{n}\left(\gamma^{*}(n), 2 \lambda\right) \geq\left(\widetilde{n}\left(\gamma^{*}\left(N_{\varepsilon}\right), 2 \lambda_{\gamma}\right)\right.$. That is, if $\left(x_{s}\right)_{s \in \mathbb{N}} \in$ $\mathscr{A}_{\xi_{\gamma}}$, then

$$
d\left(x_{\widetilde{n}\left(\gamma^{*}(n), 2 \lambda\right)}, x_{s}\right)<\varepsilon
$$

for each $s \geq s_{\varepsilon}$ for some $s_{\varepsilon} \in \mathbb{N}$. Indeed, fix a $\lambda \in \mathbb{N}$ such that $\Lambda\left(N_{\varepsilon}\right)<\gamma<2 \lambda_{\gamma}<2 \lambda-1<2 \lambda$. Then, from (25) which is valid for $n(2 \lambda)=\widetilde{n}\left(\gamma^{*}(n), 2 \lambda\right)$ we have

$$
\left(x_{\widetilde{n}\left(\gamma^{*}(n), 2 \lambda\right)}, x_{n(2 \lambda-1)}\right)<\frac{1}{3 N_{\varepsilon}}
$$

for each $n(2 \lambda-1) \geq n_{0}(2 \lambda-1)$. Now, since $\Lambda\left(N_{\varepsilon}\right)<\gamma<2 \lambda-1$ we have

$$
\left(x_{n(2 \lambda-1)}, x_{s}\right)<\frac{1}{3 N_{\varepsilon}}
$$

for some $n(2 \lambda-1)$, s onwards. On the other hand, since $\left(x_{s}\right)_{s \in \mathbb{N}}$ is right $K$-Cauchy sequence we have

$$
\left(x_{s}, x_{s^{\prime}}\right)<\frac{1}{3 N_{\varepsilon}}
$$


for $s \geq s^{\prime} \geq s_{\varepsilon}$. Finally, by combining the last three inequalities, we obtain

$$
d\left(x_{\widetilde{n}\left(\gamma^{*}(n), 2 \lambda\right)}, x_{s}\right)<\frac{1}{3 N_{\varepsilon}}+\frac{1}{3 N_{\varepsilon}}+\frac{1}{3 N_{\varepsilon}}<\frac{1}{N_{\varepsilon}}<\varepsilon
$$

for each $s \geq s_{\varepsilon}$. The last conclusion confirms the truth of (51).

To prove that $\left(\xi_{\gamma}\right)_{\gamma \in \mathbb{N}}$ is right $\widehat{d}$-cofinal to $\phi\left(x_{\widetilde{n}\left(\gamma^{*}(n), 2 \lambda\right)}\right)_{\tilde{n}\left(\gamma^{*}(n), 2 \lambda\right) \in \mathbb{N}}$, fix an $\varepsilon>0$. We define $n_{\varepsilon}=$ $\min \{n \mid 2 / n<\varepsilon\}$. Now, let $\widetilde{n}\left(\gamma^{*}(n), 2 \lambda\right) \geq \widetilde{n}\left(\gamma^{*}\left(n_{\varepsilon}\right), \Lambda\left(n_{\varepsilon}\right)\right)$ and let $\gamma_{\tilde{n}\left(\gamma^{*}(n), 2 \lambda\right)}=2 \lambda+2$. We prove that

$$
\widehat{d}\left(\xi_{\gamma}, \phi\left(x_{\tilde{n}\left(\gamma^{*}(n), 2 \lambda\right)}\right)\right)<\frac{1}{3 n}
$$

for each $\gamma>\gamma_{\widetilde{n}\left(\gamma^{*}(n), 2 \lambda\right)}$. Indeed, let $\left(x_{s}\right)_{s \in \mathbb{N}} \in \mathscr{A}_{\xi_{\gamma}}$ and $\left(x_{t}\right)_{t \in \mathbb{N}} \in \mathscr{A}_{\phi\left(\widetilde{n}\left(\gamma^{*}(n), 2 \lambda\right)\right)}$. Since

$$
\widehat{d}\left(\xi_{\gamma}, \xi_{2 \lambda-1}\right)<\frac{1}{3 n_{\varepsilon}}, \quad \widehat{d}\left(\xi_{2 \lambda-1}, \xi_{2 \lambda}\right)<\frac{1}{3 n_{\varepsilon}}
$$

using the same logic as above, we conclude that

$$
d\left(x_{s}, x_{\widetilde{n}\left(\gamma^{*}(n), 2 \lambda\right)}\right)<\frac{1}{n_{\varepsilon}}
$$

for each $s \geq s_{\varepsilon}$ for some $s_{\varepsilon} \in \mathbb{N}$. On the other hand, since $x_{t} \rightarrow x_{\tilde{n}\left(\gamma^{*}(n), 2 \lambda\right)}$ we have

$$
d\left(x_{\widetilde{n}\left(\gamma^{*}(n), 2 \lambda\right)}, x_{t}\right)<\frac{1}{n_{\varepsilon}}
$$

whenever $t \geq t_{\varepsilon}$ for some $t_{\varepsilon} \in \mathbb{N}$. Therefore, (59) and (60) imply that

$$
d\left(x_{s}, x_{t}\right)<d\left(x_{s}, x_{\widetilde{n}\left(\gamma^{*}(n), 2 \lambda\right)}\right)+d\left(x_{\widetilde{n}\left(\gamma^{*}(n), 2 \lambda\right)}, x_{t}\right)<\frac{2}{n_{\varepsilon}}<\varepsilon
$$

for each $s \geq s_{\varepsilon}$ and each $t \geq t_{\varepsilon}$. Finally, (61) implies (57) and therefore the required sequence $\left(x_{n}\right)_{n \in \mathbb{N}}$ of the hypothesis is the sequence $\left(x_{\widetilde{n}\left(\gamma^{*}(n), 2 \lambda\right)}\right)_{\tilde{n}\left(\gamma^{*}(n), 2 \lambda\right) \in \mathbb{N}}$.

Proposition 26. Let $(\eta)=\left(\eta_{\delta}\right)_{\delta \in \mathbb{N}}$ be a nonconstant left $K$ Cauchy sequence of $(\widehat{X}, \widehat{d})$ without last element. Then there exists a left $K$-Cauchy sequence $\left(y_{\sigma}\right)_{\sigma \in \mathbb{N}}$ of $(X, d)$ such that the sequences $\left(\eta_{\delta}\right)_{\delta \in \mathbb{N}}$ and $\left(\phi\left(y_{\sigma}\right)\right)_{\sigma \in \mathbb{N}}$ are left $\widehat{d}$-cofinal.

Theorem 27. Every quasi-pseudometric space has a $\kappa$ completion.

Proof. Let $(X, d)$ be a quasi-pseudometric space and let $\left(\xi_{\gamma}\right)_{\gamma \in \mathbb{N}}$ be a $\kappa$-Cauchy sequence in the space $(\widehat{X}, \widehat{d})$. Then, by Definition 11 , there exists a $\kappa$-cut $\widehat{\xi}=\left(\mathscr{A}_{\widehat{\xi}}, \mathscr{B}_{\widehat{\xi}}\right) \in \widehat{X}$ such that $\left(\xi_{\gamma}\right)_{\gamma \in \mathbb{N}} \in \mathscr{A}_{\widehat{\xi}}$. Suppose that $\mathscr{A}_{\widehat{\xi}}=\left\{\left(\xi_{\gamma(i)}^{i}\right)_{\gamma(i) \in \mathbb{N}} \mid i \in I\right\}$ and $\mathscr{B}_{\widehat{\xi}}=\left\{\left(\eta_{\delta(j)}^{j}\right)_{\delta(j) \in \mathbb{N}} \mid j \in J\right\}$. Then, $\left(\xi_{\gamma}\right)_{\gamma \in \mathbb{N}}=\left(\xi_{\gamma\left(i_{0}\right)}^{i_{0}}\right)_{\gamma\left(i_{0}\right) \in \mathbb{N}}$ for some $i_{0} \in I$. We prove that there exists a $\kappa$-cut $\xi$ in $(X, d)$ such that $\left(\xi_{\gamma}\right)_{\gamma \in \mathbb{N}}$ converges to $\xi$ with respect to $\tau(\widehat{d})$.

We define that $\mathscr{A}_{\xi}=\left\{\left(x_{n}\right)_{n \in \mathbb{N}} \mid\left(x_{n}\right)_{n \in \mathbb{N}}\right.$ is a right $K$-Cauchy sequence of $X$ such that $\left(\phi\left(x_{n}\right)_{n \in \mathbb{N}} \in \mathscr{A}_{\widehat{\xi}}\right\}$ and
$\mathscr{B}_{\xi}=\left\{\left(y_{\sigma}\right)_{\sigma \in \mathbb{N}} \mid\left(y_{\sigma}\right)_{\sigma \in \mathbb{N}}\right.$ is a left $K$-Cauchy sequence of $X$ such that $\left(\phi\left(y_{\sigma}\right)_{\sigma \in \mathbb{N}} \in \mathscr{B}_{\widehat{\xi}}\right\}$.

We first verify that $\xi=\left(\mathscr{A}_{\xi}, \mathscr{B}_{\xi}\right)$ constitute a $\kappa$-cut in $(X, d)$. For this we need to show that

(A) the classes $\mathscr{A}_{\xi}, \mathscr{B}_{\xi}$ are nonvoid,

(B) the pair $\left(\mathscr{A}_{\xi}, \mathscr{B}_{\xi}\right)$ satisfies the conditions of Definition 8.

We first show (A). We have two cases to consider.

Case A.1. There exists $\tilde{i} \in I$ such that the sequence $\left(\xi_{\gamma(\tilde{i})}^{\tilde{i}}\right)_{\gamma(\tilde{i}) \in \mathbb{N}}$ is nonconstant. In this case, Proposition 25 implies that there is a right $K$-Cauchy sequence $\left(x_{n}\right)_{n \in \mathbb{N}}$ of $X$ such that $\left(\xi_{\gamma(\tilde{i})}^{\tilde{i}}\right)_{\gamma(\tilde{i}) \in \mathbb{N}}$ and $\left(\phi\left(x_{n}\right)\right)_{n \in \mathbb{N}}$ are right $\widehat{d}$-cofinal. Therefore, $\left(\phi\left(x_{n}\right)\right)_{n \in \mathbb{N}} \in$ $\mathscr{A}_{\bar{\xi}}$ which implies that $\left(x_{n}\right)_{n \in \mathbb{N}} \in \mathscr{A}_{\xi}$.

Case A.2. For each $i \in I$, there exists $\widehat{\gamma}(i) \in \mathbb{N}$ such that $\xi_{\gamma(i)}^{i}=\xi_{\gamma(i)+1}^{i}$ whenever $\gamma(i) \geq \widehat{\gamma}(i)$. Fix an $i^{*} \in I$. Suppose that $\left(x_{n}\right)_{n \in \mathbb{N}} \in \mathscr{A}_{\xi_{\hat{\gamma}\left(i^{*}\right)}}$. Then, from $\lim _{\delta(j), \gamma\left(i^{*}\right)} \widehat{d}\left(\eta_{\delta(j)}^{j}, \xi_{\gamma\left(i^{*}\right)}^{i^{*}}\right)=0$, $\widehat{d}\left(\xi_{\gamma\left(i^{*}\right)}^{i^{*}}, \xi_{\widehat{\gamma}\left(i^{*}\right)}^{i^{*}}\right)=0$, and $\lim _{n} \widehat{d}\left(\xi_{\widehat{\gamma}\left(i^{*}\right)}^{i^{*}}, \phi\left(x_{n}\right)\right)=0$, we conclude that $\lim _{\delta(j), n} \widehat{d}\left(\eta_{\delta(j)}^{j}, \phi\left(x_{n}\right)\right)=0$. It follows that $\left(x_{n}\right)_{n \in \mathbb{N}} \in \mathscr{A}_{\xi}$. Therefore, in any case we have that $\mathscr{A}_{\xi} \neq \emptyset$. Similarly we prove that $\mathscr{B}_{\xi} \neq \emptyset$.

To show (B), we first prove the validity of Condition (i) of Definition 8. Let $\left(x_{n}\right)_{n \in \mathbb{N}} \in \mathscr{A}_{\xi}$ and $\left(y_{\sigma}\right)_{\sigma \in \mathbb{N}} \in \mathscr{B}_{\xi}$. Then, by construction of $\mathscr{A}_{\xi}$ and $\mathscr{B}_{\xi}$, we have $\lim _{\sigma, n} \widehat{d}\left(\phi\left(y_{\sigma}\right), \phi\left(x_{n}\right)\right)=$ 0 . Hence, Proposition 22 implies that $\lim _{\sigma, n} d\left(y_{\sigma}, x_{n}\right)=0$. To prove that $\xi$ satisfies the second condition of Definition 8, let $\left(x_{n}\right)_{n \in \mathbb{N}}$ and $\left(x_{m}\right)_{m \in \mathbb{N}}$ be two right $K$-Cauchy sequences of $\mathscr{A}_{\xi}$. Since $\left(\phi\left(x_{n}\right)\right)_{n \in \mathbb{N}}$ and $\left(\phi\left(x_{m}\right)\right)_{m \in \mathbb{N}}$ belong to $\mathscr{A}_{\hat{\xi}}$, it follows by definition that they are right $\widehat{d}$-cofinal. Hence, Proposition 22 implies that $\left(x_{n}\right)_{n \in \mathbb{N}}$ and $\left(x_{m}\right)_{m \in \mathbb{N}}$ are right $d$-cofinal. Clearly, the maximality of $\mathscr{A}_{\bar{\xi}}$ and $\mathscr{B}_{\bar{\xi}}$ implies the maximality of $\mathscr{A}_{\xi}$ and $\mathscr{B}_{\xi}$, respectively. Hence, condition (iii) is satisfied.

It remains to prove that $\left(\xi_{\gamma}\right)_{\gamma \in \mathbb{N}}$ converges to $\xi$ with respect to $\tau(\widehat{d})$. If $\left(\xi_{\gamma}\right)_{\gamma \in \mathbb{N}}$ is constant, then, $\xi_{\gamma}=\xi$ for some $\tilde{\gamma} \in \mathbb{N}$ onwards. Hence, by the Definition 17, we have that $\lim _{\gamma} \widehat{d}\left(\xi, \xi_{\gamma}\right)=0$. If the sequence $\left(\xi_{\gamma}\right)_{\gamma \in \mathbb{N}}$ is nonconstant, then there exists a right $K$-Cauchy sequence $\left(x_{n}\right)_{n \in \mathbb{N}} \in \mathscr{A}_{\xi}$ such that $\left(\phi\left(x_{n}\right)\right)_{t \in \mathbb{N}}$ and $\left(\xi_{\gamma}\right)_{\gamma \in \mathbb{N}}$ are right $\widehat{d}$-cofinal. Since $\left(x_{n}\right)_{n \in \mathbb{N}} \in \mathscr{A}_{\xi}$, Proposition 23 implies that $\phi\left(x_{n}\right) \rightarrow \xi$. So, by Corollary 7 we conclude that $\xi_{\gamma} \rightarrow \xi$. This completes the proof.

Definition 28. Let $(X, d)$ be a $T_{0}$-quasi-pseudometric space. We define an equivalence relation $\approx$ on $\widehat{X}=\bar{X} \cup \phi(X)$ as follows: $\xi \approx \xi^{\prime}$ if and only if $\mathscr{A}_{\xi}=\mathscr{A}_{\xi^{\prime}}$ whenever $\xi, \xi^{\prime} \in \bar{X}$ or $\xi=\phi_{i}(x)$ and $\xi^{\prime}=\phi_{j}(x)$ for some $i, j \in I_{x}$ whenever $\xi, \xi^{\prime} \in \Phi(x), x \in X$ (see Definition 9). In the following $\phi_{q}$ : $\widehat{X} \rightarrow \widetilde{X}$ will denote the isometric quotient map with respect to $\approx$ previously defined. That is, $\xi$ corresponds to $\phi_{q}(\xi)=\Xi$ whenever $\xi \in \widehat{X}$. Clearly, $\phi_{q}$ is surjective. $\widetilde{X}$ will denote the set of all equivalence classes $\Xi$. 
Proposition 29. Let $(X, d)$ be a $T_{0}$-quasi-pseudometric space and let $\widehat{X}, \widetilde{X}$ be as above. Suppose that $\widetilde{d}: \widetilde{X} \times \widetilde{X} \rightarrow \mathbb{R}$ is a function mapping defined by $\widetilde{d}\left(\Xi, \Xi^{\prime}\right)=\widehat{d}\left(\xi, \xi^{\prime}\right)$ whenever $\xi, \xi^{\prime} \in \widehat{X}$. Then, $\widetilde{d}$ determines a $T_{0}$-quasi-pseudometric on $\widetilde{X}$.

Proof. Clearly, $\approx$ is an equivalence relation on $X$. To prove that $\widetilde{d}$ is well-defined suppose that $\xi, \xi_{1}, \xi^{\prime}, \xi_{1}^{\prime} \in \widehat{X}$ and $\xi \approx \xi^{\prime}$ and $\xi_{1} \approx \xi_{1}^{\prime}$. By the triangle inequality we see that $\widehat{d}\left(\xi_{1}, \xi_{1}^{\prime}\right) \leq$ $\widehat{d}\left(\xi_{1}, \xi\right)+\widehat{d}\left(\xi, \xi^{\prime}\right)+\widehat{d}\left(\xi^{\prime}, \xi_{1}^{\prime}\right)$ and hence $\widehat{d}\left(\xi_{1}, \xi_{1}^{\prime}\right) \leq 0+$ $\widehat{d}\left(\xi, \xi^{\prime}\right)+0$. Similarly, $\widehat{d}\left(\xi, \xi^{\prime}\right) \leq \widehat{d}\left(\xi_{1}, \xi_{1}^{\prime}\right)$ which implies that $\widehat{d}\left(\xi, \xi^{\prime}\right)=\widehat{d}\left(\xi_{1}, \xi_{1}^{\prime}\right)$. Hence, $\widetilde{d}$ is well defined. It is obvious that $\widetilde{d}$ is quasi-pseudometric. To prove that $(\widetilde{X}, \widetilde{d})$ is a $T_{0}$-quasipseudometric space suppose that $\widetilde{d}\left(\Xi, \Xi^{\prime}\right)=\widetilde{d}\left(\Xi^{\prime}, \Xi\right)=0$. Then, $\widehat{d}\left(\xi, \xi^{\prime}\right)=\widehat{d}\left(\xi^{\prime}, \xi\right)=0$. Suppose that $\xi, \xi^{\prime} \in \bar{X}$. Let $\left(x_{n}\right)_{n \in \mathbb{N}} \in \mathscr{A}_{\xi}$ and $\left(x_{m}^{\prime}\right)_{m \in \mathbb{N}} \in \mathscr{A}_{\xi^{\prime}}$. Then, by Proposition 18 we conclude that $\left(x_{n}\right)_{n \in \mathbb{N}}$ and $\left(x_{m}^{\prime}\right)_{m \in \mathbb{N}}$ are right $d$-cofinal. Therefore, $\mathscr{A}_{\xi}=\mathscr{A}_{\xi^{\prime}}$. If $\xi=\phi(x)$ and $\xi^{\prime}=\phi\left(x^{\prime}\right)$ for some $x, x^{\prime} \in X$, then $\widehat{d}\left(\phi(x), \phi\left(x^{\prime}\right)\right)=\hat{d}\left(\phi\left(x^{\prime}\right), \phi(x)\right)=0$ which implies that $d\left(x, x^{\prime}\right)=d\left(x^{\prime}, x\right)=0$. Since $(X, d)$ is $T_{0}$ we conclude that $x=x^{\prime}$. Hence, in any case we have $\Xi=\Xi^{\prime}$. Consequently, $(\widetilde{X}, \widetilde{d})$ is a $T_{0}$-quasi-pseudometric space.

Proposition 30. If $(X, d)$ is a $T_{0}$-quasi-pseudometric space, then $\varphi=\phi_{q} \circ \phi:(X, d) \rightarrow(\widetilde{X}, \widetilde{d})$ is an isometric embedding.

Proof. We have that $\varphi=\phi_{q} \circ \phi$ is an isometry, since both $\phi_{q}$ and $\phi$ are isometries. On the other hand, for any $x, y \in X$, $\phi(x)=\phi(y)$ implies that $\widehat{d}(\phi(x), \phi(y))=0=\widehat{d}(\phi(y), \phi(x))$. Then, Proposition 22 implies that $d(x, y)=0=d(y, x)$. Hence, $x=y$ because $(X, d)$ is a $T_{0}$-quasi-pseudometric space. Therefore, $\phi$ is injective which implies that $\varphi$ is an isometric embedding.

Theorem 31. Let $(X, d)$ be a $T_{0}$-quasi-pseudometric space. Then $(\widetilde{X}, \widetilde{d})$ is a $T_{0} \kappa$-completion of $(X, d)$.

Proof. Let $\left(\Xi_{\gamma}\right)_{\gamma \in \mathbb{N}}$ be a $\kappa$-Cauchy sequence in $(\widetilde{X}, \widetilde{d})$. Therefore, since $\phi_{q}$ is a surjective isometry, $\phi_{q}^{-1}\left(\left(\Xi_{\gamma}\right)_{\gamma \in \mathbb{N}}\right)$ is a $\kappa$-Cauchy sequence in $(\widehat{X}, \widehat{d})$. Thus for some $\xi \in \widehat{X}$, $\phi_{q}^{-1}\left(\left(\Xi_{\gamma}\right)_{\gamma \in \mathbb{N}}\right)$ converges to $\xi$. It follows that $\left(\Xi_{\gamma}\right)_{\gamma \in \mathbb{N}}$ converges to $\phi_{q}(\xi)=\Xi$ since $\phi_{q}$ is an isometry. So $(\widetilde{X}, \widetilde{d})$ is $\kappa$-complete $T_{0}$-quasi-pseudometric space (see Proposition 29). It remains to prove that the set $\varphi(X)=\left(\phi_{q} \circ \phi\right)(X)$ is dense in $(\widetilde{X}, \widetilde{d})$. Indeed, suppose that $\Xi \in \widetilde{X}$. Then, since $\phi_{q}$ is a surjective isometry, there exists $\xi \in \widehat{X}$ such that $\phi_{q}(\xi)=\Xi$. Let $\left(x_{n}\right)_{n \in \mathbb{N}} \in \mathscr{A}_{\xi}$. Then, by Definition 17 we have that $\phi\left(x_{n}\right) \rightarrow$ $\xi$. Therefore, $\phi_{q}\left(\phi\left(x_{n}\right)\right)$ converges to $\phi_{q}(\xi)=\Xi$ with respect to $\tau_{\tilde{d}}$. It follows that $\varphi(X)$ is dense in $(\widetilde{X}, \widetilde{d})$ which implies that $(\widetilde{X}, \widetilde{d})$ is a $\kappa$-completion of $(X, d)$ (see Definition 16$)$.

Definition 32. Let $(X, d)$ be a $T_{0}$-quasi-pseudometric space. Then the $T_{0}$-quasi-pseudometric space $(\widetilde{X}, \widetilde{d})$ previously defined will be called the standard $\kappa$-completion of $(X, d)$.

\section{A Categorical View of the $\kappa$-Completion}

As already pointed out, a natural problem which arises in quasi-pseudometric spaces is to introduce a convenient notion of completeness and to construct a satisfactory theory of completion. What do we mean by a "satisfactory theory" of completeness and completion for quasi-pseudometric spaces? According to Doitchinov, the answer to that question is based on certain restrictions that we must have. These restrictions are separated in two groups as follows.

$(\alpha)$ A notion of Cauchy sequence in any quasipseudometric space has to be defined in such a manner that the following requirements are fulfilled:

(i) every convergent sequence is a Cauchy sequence;

(ii) when $(X, d)$ is a pseudometric space, the Cauchy sequences are the usual ones.

( $\beta$ ) A standard construction of a completion $(\widehat{X}, \widehat{d})$ of any quasi-pseudometric space $(X, d)$ may need the following.

(iii) If $(X, d) \subseteq(Y, \rho)$, then $(\widehat{X}, \widehat{d}) \subseteq(\widehat{Y}, \widehat{\rho})$, where inclusions are understood as quasimetric embeddings (an embedding between two objects $X$ and $Y$ is an injective (one-to-one) and structure-preserving map $f: X \rightarrow$ $Y$. A structure on a set consists of additional mathematical objects (topological structures, orders, and equivalence relations) that in some manner attach to the set. The precise meaning of "structure-preserving" depends on the kind of mathematical structure of which $X$ and $Y$ are instances. In general topology, an embedding is a homeomorphism or topological isomorphism (a bicontinuous function between two topological spaces) onto its image. More explicitly, a map $f: X \rightarrow Y$ between topological spaces $X$ and $Y$ is an embedding if $f$ yields a homeomorphism between $X$ and $f(X)$ (where $f(X)$ carries the subspace topology inherited from $Y$ ). In quasimetric spaces such homeomorphisms are called isometries or quasimetric embeddings.) and the second one is an extension of the former.

(iv) In the case when $(X, d)$ is a metric space, $(\widehat{X}, \widehat{d})$ is the usual metric completion of $(X, d)$.

After the paper of Künzi and Schellekens [15] another requirement must be added in $(\beta)$. This is the following.

(v) The constructed completion must be an idempotent operation; that is, the $\kappa$-completion of the $\kappa$-completion of $(X, d)$ is isomorphic to the $\kappa$-completion of $(X, d)$.

The $\kappa$-completion differs with all other previously introduced completion theories for quasi-pseudometric spaces. 
More precisely, in metric spaces, symmetry enables us to construct the classical completion by using Cauchy sequences instead of equivalence classes of Cauchy sequences. Similarly in the $\kappa$-procedure, since right (resp. left) $d$-cofinality between right $K$-Cauchy sequences (resp. left $K$-Cauchy cosequences) is an equivalence relation, we use Cauchy sequences instead of $\kappa$-cuts. On the other hand, in metric spaces, the notions of Cauchy sequence, right $K$-Cauchy sequence, and left $K$-Cauchy cosequence coincide. Then, in the $\kappa$-procedure, every class of equivalent Cauchy sequences of the classical case, identifies with the class of right $K$-Cauchy sequences of a $\kappa$-cut and vice versa. We now show that the notion of $\kappa$-completeness and the constructed $\kappa$-completion satisfy the requirements posed by Doitchinov [1, page 128$]$ as well as the requirement (v) previously posed.

The following two propositions show that the $\kappa$ completion satisfies the requirements (i) and (ii) previously stated.

Proposition 33. Every convergent right $K$-Cauchy sequence is a $\kappa$-Cauchy sequence.

Proof. In fact, if a right $K$-Cauchy sequence $\left(x_{n}\right)_{n \in \mathbb{N}}$ converges to a point $x$ of $X$, then it belongs to the first class of a $\kappa$-cut $\phi_{i}(x) \in \Phi(x)$. The validity of this proposition follows from Definition 11.

Clearly, in the case of metric spaces, the notions of converging right $K$-Cauchy sequence and converging sequence coincide.

The following proposition is obvious.

Proposition 34. If $(X, d)$ is a pseudometric space, then the notion of $\kappa$-Cauchy sequence coincides with the classical one.

Remark 35. Doitchinov in [1, Proposition 1] shows that if a sequence $\left(x_{n}\right)_{n \in \mathbb{N}}$ converges to a point, say $x$, then $\left(x_{n}\right)_{n \in \mathbb{N}}$ has $x$ as its cosequence, so it is a $D$-Cauchy sequence. (A sequence $\left(x_{n}\right)_{n \in \mathbb{N}}$ in the quasimetric space $(X, d)$ is called $D$ Cauchy sequence provided that for any $\kappa \in \mathbb{N}$ there exists a $y_{\kappa} \in X$ and $N_{\kappa} \in \mathbb{N}$ such that $d\left(y_{m}, x_{n}\right)<1 / \kappa$ when $m, n>N_{\kappa}$.) In another point in [1, page 130], he also says that a "good" $D$-Cauchy sequence is that which remains $D$ Cauchy sequence even if we withdraw the limit point from the space. We recall his counterexample as follows: let $(X, d)$ be a quasimetric space, $X=\left\{x_{n} \mid n=0,1,2, \ldots\right\}$ and

$$
d\left(x_{n}, x_{m}\right)= \begin{cases}1 & \text { for } m>0, m \neq n \\ \frac{1}{n} & \text { for } m=0, n \neq 0 \\ 0 & \text { for } m=n\end{cases}
$$

The sequence $\left(x_{n}\right)_{n \in \mathbb{N}}$ converges to $x_{0}$, so it must be a $D$ Cauchy sequence. However, if we withdraw the limit point $x_{0}$, the space becomes a discrete metric space, so $\left(x_{n}\right)_{n \in \mathbb{N}}$ ceases to be a $D$-Cauchy sequence. Of course, this conclusion contradicts with Doitchinov's first aspect. It is easy to see that with the notion of $\kappa$-Cauchy sequence we avoid this undesirable situation. In fact, $\left(x_{n}\right)_{n \in \mathbb{N}}$ is not a $\kappa$-Cauchy sequence.
The requirement (iii) of Doitchinov concerns the categorical aspect of a completion. It is well known that the class of $D$ complete quasimetric spaces (a quasimetric space is called $D$ complete if every $D$-Cauchy sequence is convergent) defines a category $\mathbb{Q} \mathscr{M} \mathcal{S}$ provided that we assume as morphisms the quasiuniformly continuous functions. Bonsangue et al. [16] defines the category $\mathscr{G} \mathscr{M} \mathcal{S}$ with complete generalized metric spaces (a generalized metric space consists of a set $X$ together with a distance function $d: X \times X \rightarrow[0, \infty]$, satisfying $d(x, x)=0$ and $d(x, y)<d(x, z)+d(z, y)$, for all $x, z$, and $y$ in $X$. We remark that traditionally a quasi-pseudometric space is required to take finite values. However the Yoneda completion of [16] involves generalized metric which allows for infinite distances) as objects and nonexpansive maps (a function $f$ between two quasi-pseudometric spaces $(X, d)$ and $(Y, \rho)$ is non-expansive provided that $\rho(f(x), f(y)) \leq$ $d(x, y)$ for all $x, y \in X$.) as morphisms.

We define a new category $Q \mathscr{P} \mathscr{M} \mathcal{S}$ whose objects are the $T_{0}$ quasi-pseudo-metric spaces and morphisms are the $\kappa$-cut preserving functions defined below (Definition 39).

Definition 36. A function $f$ between two $T_{0}$ quasipseudometric spaces $(X, d)$ and $(Y, \rho)$ is called quasiuniformly continuous if for any $\epsilon>0$ there is a $\delta>0$ such that $\rho\left(x, x^{\prime}\right)<\epsilon$ whenever $d\left(x, x^{\prime}\right)<\delta$.

Definition 37. Two sequences $\left(x_{n}\right)_{n \in \mathbb{N}}$ and $\left(y_{\sigma}\right)_{\sigma \in \mathbb{N}}$ in a $T_{0}$ quasi-pseudometric space $(X, d)$ form a $\kappa$-Cauchy pair of sequences if there exists a $\kappa$-cut $\xi=(\mathscr{A}, \mathscr{B})$ in $X$ such that $\left(x_{n}\right)_{n \in \mathbb{N}} \in \mathscr{A}$ and $\left(y_{\sigma}\right)_{\sigma \in \mathbb{N}} \in \mathscr{B}$. Two $\kappa$-Cauchy pairs of sequences are $\kappa$-compatible if they belong to the same $\kappa$-cut.

Definition 38. Let $(X, d),(Y, \rho)$ be $T_{0}$ quasi-pseudometric spaces and let $f: X \rightarrow Y$ be a continuous function. Let also $\left(\left(x_{n}\right)_{n \in \mathbb{N}},\left(y_{\sigma}\right)_{\sigma \in \mathbb{N}}\right)$ be a $\kappa$-Cauchy pair of sequences in $(X, d)$. We say that a $\kappa$-Cauchy pair $\left(\left(x_{m}\right)_{m \in \mathbb{N}},\left(y_{s}\right)_{s \in \mathbb{N}}\right)$ of sequences in $(X, d)$ is $(\kappa, f)$-compatible to $\left(\left(x_{n}\right)_{n \in \mathbb{N}},\left(y_{\sigma}\right)_{\sigma \in \mathbb{N}}\right)$ if it satisfies the following conditions:

(i) $\left(\left(x_{n}\right)_{n \in \mathbb{N}},\left(y_{\sigma}\right)_{\sigma \in \mathbb{N}}\right)$ and $\left(\left(x_{m}\right)_{m \in \mathbb{N}},\left(y_{s}\right)_{s \in \mathbb{N}}\right)$ are $\kappa-$ compatible;

(ii) $\left(\left(f\left(x_{m}\right)\right)_{m \in \mathbb{N}}, \quad\left(f\left(y_{s}\right)\right)_{s \in \mathbb{N}}\right)$ is a $\kappa$-Cauchy pair of sequences in $Y$.

Definition 39. Let $(X, d),(Y, \rho)$ be $T_{0}$ quasi-pseudometric spaces. A continuous function $f: X \rightarrow Y$ is called $\kappa$-cut preserving if it satisfies the following conditions.

(i) For each $\kappa$-Cauchy pair $\left(\left(x_{n}\right)_{n \in \mathbb{N}},\left(y_{\sigma}\right)_{\sigma \in \mathbb{N}}\right)$ of sequences in $X$, there exists a $\kappa$-Cauchy pair $\left(\left(x_{m}\right)_{m \in \mathbb{N}},\left(y_{s}\right)_{s \in \mathbb{N}}\right)$ of sequences in $X$ such that $\left(\left(x_{m}\right)_{m \in \mathbb{N}},\left(y_{s}\right)_{s \in \mathbb{N}}\right)$ is $(\kappa, f)$-compatible to $\left(\left(x_{n}\right)_{n \in \mathbb{N}}\right.$, $\left.\left(y_{\sigma}\right)_{\sigma \in \mathbb{N}}\right)$.

(ii) If $\left(\left(x_{n}\right)_{n \in \mathbb{N}},\left(y_{\sigma}\right)_{\sigma \in \mathbb{N}}\right),\left(\left(x_{n}^{\prime}\right)_{n \in \mathbb{N}},\left(y_{\sigma}^{\prime}\right)_{\sigma \in \mathbb{N}}\right), \quad\left(\left(x_{m}\right)_{m \in \mathbb{N}}\right.$, $\left.\left(y_{s}\right)_{s \in \mathbb{N}}\right)$ and $\left(\left(x_{m}^{\prime}\right)_{m \in \mathbb{N}},\left(y_{s}^{\prime}\right)_{s \in \mathbb{N}}\right)$ are $\kappa$-Cauchy pairs of sequences such that $\left(\left(x_{m}\right)_{m \in \mathbb{N}},\left(y_{s}\right)_{s \in \mathbb{N}}\right)$ is $(\kappa, f)$ compatible to $\left(\left(x_{n}\right)_{n \in \mathbb{N}},\left(y_{\sigma}\right)_{\sigma \in \mathbb{N}}\right)$ and $\left(\left(x_{m}^{\prime}\right)_{m \in \mathbb{N}}\right.$, $\left.\left(y_{s}^{\prime}\right)_{s \in \mathbb{N}}\right)$ is $(\kappa, f)$-compatible to $\left(\left(x_{n}^{\prime}\right)_{n \in \mathbb{N}},\left(y_{\sigma}^{\prime}\right)_{\sigma \in \mathbb{N}}\right)$, then, the $\kappa$-compatibility (resp. non- $\kappa$-compatibility) 
of $\left(\left(x_{n}\right)_{n \in \mathbb{N}},\left(y_{\sigma}\right)_{\sigma \in \mathbb{N}}\right)$ and $\left(\left(x_{n}^{\prime}\right)_{n \in \mathbb{N}},\left(y_{\sigma}^{\prime}\right)_{\sigma \in \mathbb{N}}\right)$ implies the $\kappa$-compatibility (non- $\kappa$-compatibility) of $\left(\left(f\left(x_{m}\right)\right)_{m \in \mathbb{N}},\left(f\left(y_{s}\right)\right)_{s \in \mathbb{N}}\right) \quad$ and $\quad\left(\left(f\left(x_{m}^{\prime}\right)\right)_{m \in \mathbb{N}}\right.$, $\left.\left(f\left(y_{s}^{\prime}\right)\right)_{s \in \mathbb{N}}\right)$, respectively.

By the previously mentioned definition, it is clear that a cut preserving function $f$ takes distinct elements of $X$ to distinct elements of $Y$. On the other hand, a cut preserving cut $f$ is one that "embeds" $X$ as a subset of $Y$. In view of the Definitions 8, 11, and 37, Proposition 22, and Theorem 27, one obtains that the quasi-pseudometric embedding $\phi$ is a $\kappa$-cut preserving function.

Doitchinov [1] uses quasiuniformly continuous functions defined in quasimetric spaces. Lemma 7 in [1] shows that these functions are $\kappa$-cut preserving functions.

Similarly to what is in the theory of metric spaces, the categorical notion of a $T_{0}$ quasi-pseudometric completion is the following: a complete $T_{0}$ quasi-pseudometric space $(\widetilde{X}, \widetilde{d})$ is called a $\kappa$-completion of a given $T_{0}$ quasi-pseudometric space $(X, d)$ if there exists a $\kappa$-cut preserving function $\varphi$ : $(X, d) \rightarrow(\widetilde{X}, \widetilde{d})$ such that

(a) $\varphi(X)$ is dense in $(\widetilde{X}, \widetilde{d})$;

(b) the quasi-pseudometric $(X, d)$ is the inverse image of $(\widetilde{X}, \widetilde{d})$ under $\varphi$;

(c) for any $\kappa$-cut preserving function $f:(X, d) \rightarrow(Y, \rho)$, where $(Y, \rho)$ is a $\kappa$-complete $T_{0}$ quasi-pseudometric space, there is a $\kappa$-cut preserving function $f^{*}$ : $(\widetilde{X}, \widetilde{d}) \rightarrow(Y, \rho)$ with $f=f^{*} \circ \varphi$.

The mapping $\varphi$ is called the canonical quasipseudometric embedding of $(X, d)$ into $(\widetilde{X}, \widetilde{d})$.

Proposition 40. Let $(X, d),(Y, \rho)$ be two $T_{0}$ quasipseudometric spaces, let $(Y, \rho)$ be $\kappa$-complete, and let $f:(X, d) \rightarrow(Y, \rho)$ be a $\kappa$-cut preserving function. Then, there exists a $\kappa$-cut preserving function $f^{*}:(\widetilde{X}, \widetilde{d}) \rightarrow(Y, \rho)$ such that $f=f^{*} \circ \varphi$.

Proof. Let $(X, d),(Y, \rho)$, and $f$ be as in the hypothesis of the proposition. Now define a mapping $f^{*}:(\widetilde{X}, \widetilde{d}) \rightarrow$ $(Y, \rho)$ by letting $f^{*}(\Xi)=\lim _{n} f\left(x_{n}\right)$ whenever $\xi$ is a $\kappa$-cut in $X$ such that $\phi_{q}(\xi)=\Xi$ and $\left(x_{n}\right)_{n \in \mathbb{N}} \in \mathscr{A}_{\xi}$. Definitions 28 and 39 imply that $f^{*}$ takes distinct elements of $\widetilde{X}$ to distinct elements of $Y$. Let $\Xi=\varphi(x)$ where $\varphi=\phi_{q} \circ$ $\phi:(X, d) \rightarrow(\widetilde{X}, \widetilde{d})$ be the isometric embedding defined in Proposition 30. Then, by the continuity of $f$ at $x$, we have $f^{*}(\varphi(x))=f(x)$. It remains to prove that $f^{*}$ is $\kappa$ cut preserving. Let $\left(\left(\Xi_{\gamma}\right)_{\gamma \in \mathbb{N}},\left(H_{\beta}\right)_{\beta \in \mathbb{N}}\right)$ be a pair Cauchy of sequences in $\widetilde{X}$. Suppose that for each $\gamma, \beta \in \mathbb{N}$ we have $\Xi_{\gamma}=$ $\phi_{q}\left(\xi_{\gamma}\right)$ and $H_{\beta}=\phi_{q}\left(\eta_{\beta}\right)$. Then, let $\left(\left(\xi_{\gamma}\right)_{\gamma \in \mathbb{N}},\left(\eta_{\beta}\right)_{\beta \in \mathbb{N}}\right)$ be a pair Cauchy of sequences in $\widehat{X}$. Then, by Propositions 25 and 26 and Theorem 27 there is a pair Cauchy $\left(\left(x_{n}\right)_{n \in \mathbb{N}},\left(y_{\sigma}\right)_{\sigma \in \mathbb{N}}\right)$ of sequences in $X$ such that $\left(\left(\xi_{\gamma}\right)_{\gamma \in \mathbb{N}},\left(\eta_{\beta}\right)_{\beta \in \mathbb{N}}\right)$ and $\left(\left(\phi\left(x_{n}\right)\right)_{n \in \mathbb{N}}\right.$, $\left.\left(\phi\left(y_{\sigma}\right)\right)_{\sigma \in \mathbb{N}}\right)$ are $\kappa$-compatible, $(\xi)_{\gamma \in \mathbb{N}}$ and $\left(\left(\phi\left(x_{n}\right)\right)_{n \in \mathbb{N}}\right.$ are right $\widehat{d}$-cofinal, and $\left(\eta_{\beta}\right)_{\beta \in \mathbb{N}}$ and $\left(\left(\phi\left(y_{\sigma}\right)\right)_{\sigma \in \mathbb{N}}\right.$ are left $\widehat{d}$-cofinal. Since $f$ is cut preserving, there exists a Cauchy pair $\left(\left(x_{m}\right)_{m \in \mathbb{N}}\right.$, $\left.\left(y_{s}\right)_{s \in \mathbb{N}}\right)$ of sequences satisfying the conditions (i) and (ii) of Definition 39. Since $\varphi=\phi_{q} \circ \phi$ is an isometry and $f=$ $f^{*} \circ \varphi$ it follows that $f^{*}$ satisfies the conditions (i) and (ii) of Definition 39. Hence, $f^{*}$ is $\kappa$-cut preserving.

The previous proposition shows that the standard $\kappa$ completion is the smallest $\kappa$-complete quasi-pseudometric space containing $X$ in the sense that any other $\kappa$-complete space $Y$ containing $X$ as a subspace also contains $X$ as a subspace.

The next two propositions show that the $\kappa$-completion satisfies the requirements (iii) and (iv) posed by Doitchinov.

Proposition 41. Let $(X, d),(Y, \rho)$ be two $T_{0}$ quasipseudometric spaces, and let $(\widetilde{X}, \widetilde{d}),(\widetilde{Y}, \widetilde{\rho})$ be their standard $\kappa$-completions with canonical maps $\varphi_{X}:(X, d) \rightarrow(\widetilde{X}, \tilde{d})$ and $\varphi_{Y}:(Y, \rho) \rightarrow(\tilde{Y}, \tilde{\rho})$. If $f:(X, d) \rightarrow(Y, \rho)$ is a $\kappa$-cut preserving function, then there exists a $\kappa$-cut preserving function $f^{*}:(\widetilde{X}, \widetilde{d}) \rightarrow(\widetilde{Y}, \widetilde{\rho})$.

Proof. The existence of a $\kappa$-cut preserving function $f^{*}$ : $(\widetilde{X}, \widetilde{d}) \rightarrow(\widetilde{Y}, \widetilde{\rho})$ follows from Proposition 40 when one applies it to the function $\varphi_{Y} \circ f$.

Proposition 42. If $(X, d)$ is a metric space, then the standard $\kappa$-completion $(\widetilde{X}, \widetilde{d})$ coincides with the usual metric completion.

Proof. Let $(X, d)$ be a metric space. Then in the $\kappa$-procedure the notions of right $K$-Cauchy sequence, left $K$-Cauchy cosequence, and Cauchy sequence coincide. Hence, every class of equivalent Cauchy sequences of the classical case identifies with a $\kappa$-cut and vice versa. Hence, the new elements of the two completions, the first of the classical completion and the second of the $\kappa$-completion, coincide. The rest is obvious.

Finally, let us discuss the requirement (v) previously stated in the introduction of this section. It is well known in classical metric space theory that Cauchy completion is idempotent. One can also verify that the bicompletion as well as the Doitchinov completion is an idempotent operation. The idempotency of the well known completions for quasipseudometric spaces has not been studied. There are also two main types of domain theoretic completions, the Smyth completion [17] and the Yoneda completion [16]. The Smyth completion is idempotent, while the Yoneda completion is not (see [15, page 164]). Recently, Künzi and Schellekens in [15] introduce a general net-version for the Yoneda completion and characterize the family of generalized metric spaces (generalized metric spaces are a common generalization of preorders and ordinary metric spaces. Such spaces consist of a set $X$ together with a distance function $d: X \times X \rightarrow[0, \infty]$ satisfying $d(x, x)=0$ and $d(x, z) \leq d(x, y)+d(y, z)$, for all $x, y$ and $z$ in $X$.) for which the Yoneda completion is idempotent.

We recall the classical definition of idempotency (adapted to the specific case of the $\kappa$-completion). In the following $(\widetilde{\widetilde{X}}, \widetilde{\widetilde{d}})$ denotes the $\kappa$-completion of the $\kappa$-completion $(\widetilde{X}, \widetilde{d})$. 
Definition 43. The $\kappa$-completion of a $T_{0}$ quasi-pseudometric space $(X, d)$ is idempotent if and only if there exists an isometry $f:(\widetilde{X}, \widetilde{d}) \rightarrow(\widetilde{\widetilde{X}}, \widetilde{\tilde{d}})$ such that for each $\widetilde{\Xi} \in \widetilde{\widetilde{X}}$, $\widetilde{\Xi}=f(\Xi)$ for some $\Xi \in(\widetilde{X}, \widetilde{d})$.

Proposition 44. The $\kappa$-completion is idempotent.

Proof. Let $(X, d)$ be a quasi-pseudometric space and let $(\widetilde{X}, \widetilde{d})$ be its $\kappa$-completion. Let us denote by $\widetilde{\widetilde{X}}$ the set of $\kappa$-cuts in $\widetilde{X}$ and let $\widetilde{\Xi}=\left(\mathscr{A}_{\widetilde{\Xi}}, \mathscr{B}_{\widetilde{\Xi}}\right) \in \widetilde{\widetilde{X}}$. Suppose that $\mathscr{A}_{\widetilde{\Xi}}=\left\{\left(\Xi_{\gamma(i)}^{i}\right)_{\gamma(i) \in \mathbb{N}} \mid\right.$ $i \in I\}$ and $\mathscr{B}_{\tilde{\xi}}=\left\{\left(H_{\delta(j)}^{j}\right)_{\delta(j) \in \mathbb{N}} \mid j \in J\right\}$. Then, the families $\mathscr{A}_{\widehat{\xi}}=\left\{\left(\xi_{\gamma(i)}^{i}\right)_{\gamma(i) \in \mathbb{N}} \mid i \in I\right\}$ and $\mathscr{B}_{\widehat{\xi}}=\left\{\left(\eta_{\delta(j)}^{j}\right)_{\delta(j) \in \mathbb{N}} \mid j \in J\right\}$, where $\phi_{q}\left(\xi_{\gamma(i)}^{i}\right)=\Xi_{\gamma(i)}^{i}$ and $\phi_{q}\left(\eta_{\delta(j)}^{j}\right)=H_{\delta(j)}^{j}$, define a $\kappa$-cut $\widehat{\xi}=$ $\left(\mathscr{A}_{\widehat{\xi}}, \mathscr{B}_{\widehat{\xi}}\right)$ in $\widehat{X}$. Then, according to Theorem 27 , to each $\kappa$-cut $\widehat{\xi}$ there corresponds a $\kappa$-cut $\xi \in(X, d)$. Let $\Xi=\phi_{q}(\xi)$ and let $\widetilde{\varphi}$ be the quasi-pseudometric embedding of $(\widetilde{X}, \widetilde{d})$ into $(\widetilde{\widetilde{X}}, \widetilde{\widetilde{d}})$ as it is defined in Definition 9. Then, $\widetilde{\Xi}=\widetilde{\varphi}(\Xi)$. Therefore, we have $\widetilde{\widetilde{X}}=\widetilde{\varphi}(\widetilde{X})$. Next, for each $\widetilde{\Xi}^{\prime}, \widetilde{\Xi}^{\prime \prime} \in \widetilde{\widetilde{X}}$ we have

$$
\widetilde{\widetilde{d}}\left(\widetilde{\Xi}^{\prime}, \widetilde{\Xi}^{\prime \prime}\right)=\widetilde{\widetilde{d}}\left(\widetilde{\varphi}\left(\Xi^{\prime}\right), \widetilde{\varphi}\left(\Xi^{\prime \prime}\right)\right)=\widetilde{d}\left(\Xi^{\prime}, \Xi^{\prime \prime}\right)=\widehat{d}\left(\xi^{\prime}, \xi^{\prime \prime}\right),
$$

where $\Xi^{\prime}=\phi_{q}\left(\xi^{\prime}\right)$ and $\Xi^{\prime \prime}=\phi_{q}\left(\xi^{\prime \prime}\right)$. Clearly, $\widetilde{\varphi}$ is an isometry. To complete the proof we must show that $(\widetilde{\widetilde{X}}, \widetilde{\widetilde{d}})$ is $\kappa$-complete. Indeed, let $\left(\widetilde{\Xi}_{\gamma}\right)_{\gamma \in \mathbb{N}}$ be a $\kappa$-Cauchy sequence in $(\widetilde{\widetilde{X}}, \widetilde{\widetilde{d}})$. Then, there exists a $\kappa$-cut $\widetilde{\widetilde{\Xi}}=\left(\mathscr{A}_{\tilde{\widetilde{\Xi}}}, \mathscr{B}_{\widetilde{\widetilde{\Xi}}}\right)$ in $(\widetilde{\widetilde{X}}, \widetilde{\widetilde{d}})$ such that $\left(\widetilde{\Xi}_{\gamma}\right)_{\gamma \in \mathbb{N}} \in \mathscr{A}_{\widetilde{\Xi}}$. Suppose that $\mathscr{A}_{\widetilde{\Xi}}=\left\{\left(\widetilde{\Xi}_{\gamma(i)}^{i}\right)_{\gamma(i) \in \mathbb{N}} \mid i \in I\right\}, \mathscr{B}_{\widetilde{\Xi}}=$ $\left\{\left(\widetilde{H}_{\delta(j)}^{j}\right)_{\delta(j) \in \mathbb{N}} \mid j \in J\right\}$ and $\left(\widetilde{\Xi}_{\gamma}\right)_{\gamma \in \mathbb{N}}=\left(\widetilde{\Xi}_{\gamma\left(i_{0}\right)}^{i_{0}}\right)_{\gamma\left(i_{0}\right) \in \mathbb{N}}$ for some $i_{0} \in I$. For each $i \in I$ and each $j \in J$ we have $\widetilde{\Xi}_{\gamma(i)}^{i}=\widetilde{\varphi}\left(\Xi_{\gamma(i)}^{i}\right)$ and $\widetilde{H}_{\delta(j)}^{i}=\widetilde{\varphi}\left(H_{\delta(j)}^{j}\right)$. On the other hand, $\Xi_{\gamma(i)}^{i}=\phi_{q}\left(\xi_{\gamma(i)}^{i}\right)$ and $H_{\delta(j)}^{j}=\phi_{q}\left(\eta_{\delta(j)}^{j}\right)$ for some $\xi_{\gamma(i)}^{i}, \eta_{\delta(j)}^{j} \in(X, d)$. Next, we define $\widehat{\xi}=\left(\mathscr{A}_{\widehat{\xi}}, \mathscr{B}_{\bar{\xi}}\right)$, where $\mathscr{A}_{\widehat{\xi}}=\left\{\left(\xi_{\gamma(i)}^{i}\right)_{\gamma(i) \in \mathbb{N}} \mid i \in I\right\}$ and $\mathscr{B}_{\bar{\xi}}=\left\{\left(\eta_{\delta(j)}^{i}\right)_{\delta(j) \in \mathbb{N}} \mid j \in J\right\}$. By Theorem 27, there exists a $\kappa$ cut $\xi \in(\widehat{X}, \widehat{d})$ such that, for each $\gamma(i) \in \mathbb{N},\left(\xi_{\gamma(i)}^{i}\right)_{\gamma(i) \in \mathbb{N}} \rightarrow$ $\xi$ with respect to $\tau(\widehat{d})$. By definition of $\widetilde{d}$, it follows that $\left(\Xi_{\gamma(i)}^{i}\right)_{\gamma(i) \in \mathbb{N}} \rightarrow \Xi$ with respect to $\tau(\tilde{d})$, where $\Xi=\phi_{q}(\xi)$. Since $\widetilde{\varphi}$ is an isometry, Proposition 25 implies that $\left(\widetilde{\Xi}_{\gamma(i)}^{i}\right)_{\gamma(i) \in \mathbb{N}}$ and $\left(\widetilde{\varphi}\left(\Xi_{\gamma(i)}^{i}\right)\right)_{\gamma(i) \in \mathbb{N}}$ are right $\widetilde{\widetilde{d}}$-cofinal. Therefore, Corollary 7 implies that $\left(\widetilde{\Xi}_{\gamma(i)}^{i}\right)_{\gamma(i) \in \mathbb{N}}$ converges to $\widetilde{\varphi}(\Xi)$ with respect to $\tau(\widetilde{\widetilde{d}})$. It follows that $\left(\widetilde{\Xi}_{\gamma}\right)_{\gamma \in \mathbb{N}}=\left(\widetilde{\Xi}_{\gamma\left(i_{0}\right)}^{i_{0}}\right)_{\gamma\left(i_{0}\right) \in \mathbb{N}}$ converges to $\widetilde{\varphi}(\Xi)$ with respect to $\tau(\widetilde{\widetilde{d}})$.

\section{References}

[1] D. Doitchinov, "On completeness in quasi-metric spaces," Topology and its Applications, vol. 30, no. 2, pp. 127-148, 1988.

[2] J. C. Kelly, "Bitopological spaces," Proceedings of the London Mathematical Society, vol. 13, pp. 71-89, 1963.
[3] I. L. Reilly, P. V. Subrahmanyam, and M. K. Vamanamurthy, "Cauchy sequences in quasipseudometric spaces," Monatshefte für Mathematik, vol. 93, no. 2, pp. 127-140, 1982.

[4] R. Stoltenberg, "Some properties of quasi-uniform spaces," Proceedings of the London Mathematical Society, vol. 17, no. 3, pp. 226-240, 1967.

[5] I. L. Reilly, "A generalized contraction principle," Bulletin of the Australian Mathematical Society, vol. 10, pp. 359-363, 1974.

[6] P. V. Subrahmanyam, "Remarks on some fixed-point theorems related to Banach's contraction principle," Journal of Mathematical and Physical Sciences, vol. 8, pp. 445-457, 1974.

[7] K. K. Tan, "Fixed point theorems for nonexpansive mappings," Pacific Journal of Mathematics, vol. 41, pp. 829-842, 1972.

[8] D. Doitchinov, "A concept of completeness of quasi-uniform spaces," Topology and its Applications, vol. 38, no. 3, pp. 205-217, 1991.

[9] P. Fletcher and W. Hunsaker, "A note on totally bounded quasiuniformities," Serdica, vol. 24, no. 1, pp. 95-98, 1998.

[10] H.-P. A. Künzi, "Totally bounded quiet quasi-uniformities," Topology Proceedings, vol. 15, pp. 113-115, 1990.

[11] H.-P. Künzi and C. M. Kivuvu, "A double completion for an arbitrary $T_{0}$-quasi-metric space," The Journal of Logic and Algebraic Programming, vol. 76, no. 2, pp. 251-269, 2008.

[12] H.-P. Künzi and C. M. Kivuvu, "The $B$-completion of a $T_{0}$-quasimetric space," Topology and its Applications, vol. 156, no. 12, pp. 2070-2081, 2009.

[13] H. M. MacNeille, "Partially ordered sets," Transactions of the American Mathematical Society, vol. 42, no. 3, pp. 416-460, 1937.

[14] A. Andrikopoulos and J. Stabakis, "A solution to the completion problemof quasi-uniform spaces," http://arxiv.org/abs/ 1008.1402 .

[15] H. P. Künzi and M. P. Schellekens, "On the Yoneda completion of a quasi-metric space," Theoretical Computer Science, vol. 278, no. 1-2, pp. 159-194, 2002.

[16] M. M. Bonsangue, F. van Breugel, and J. J. M. M. Rutten, "Generalized metric spaces: completion, topology, and powerdomains via the Yoneda embedding," Theoretical Computer Science, vol. 193, no. 1-2, pp. 1-51, 1998.

[17] M. B. Smyth, "Quasi-uniformities: reconciling domains with metric spaces," in Mathematical Foundations of Programming Language Semantics (New Orleans, LA, 1987), vol. 298 of Lecture Notes in Computer Science, pp. 236-253, Springer, Berlin, Germany, 1988. 


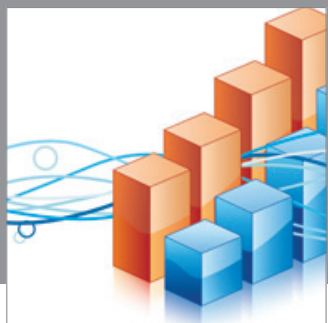

Advances in

Operations Research

mansans

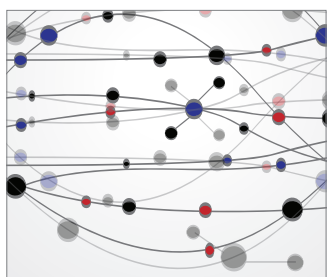

The Scientific World Journal
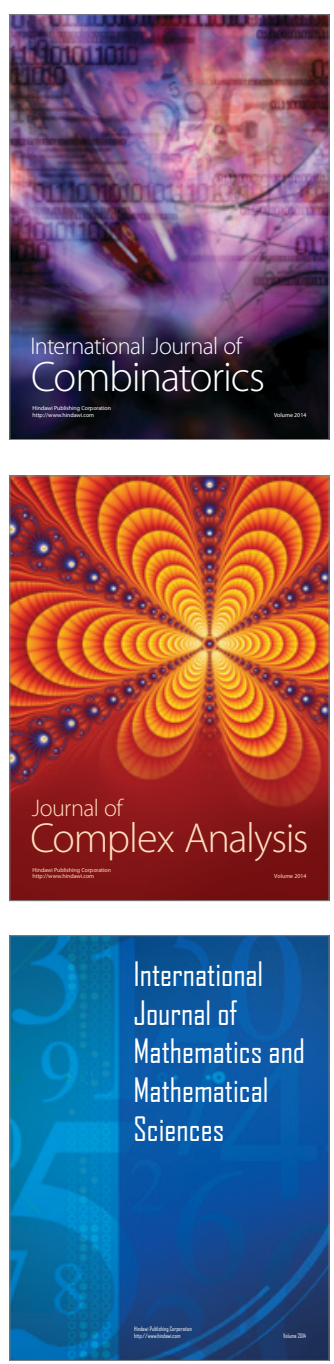
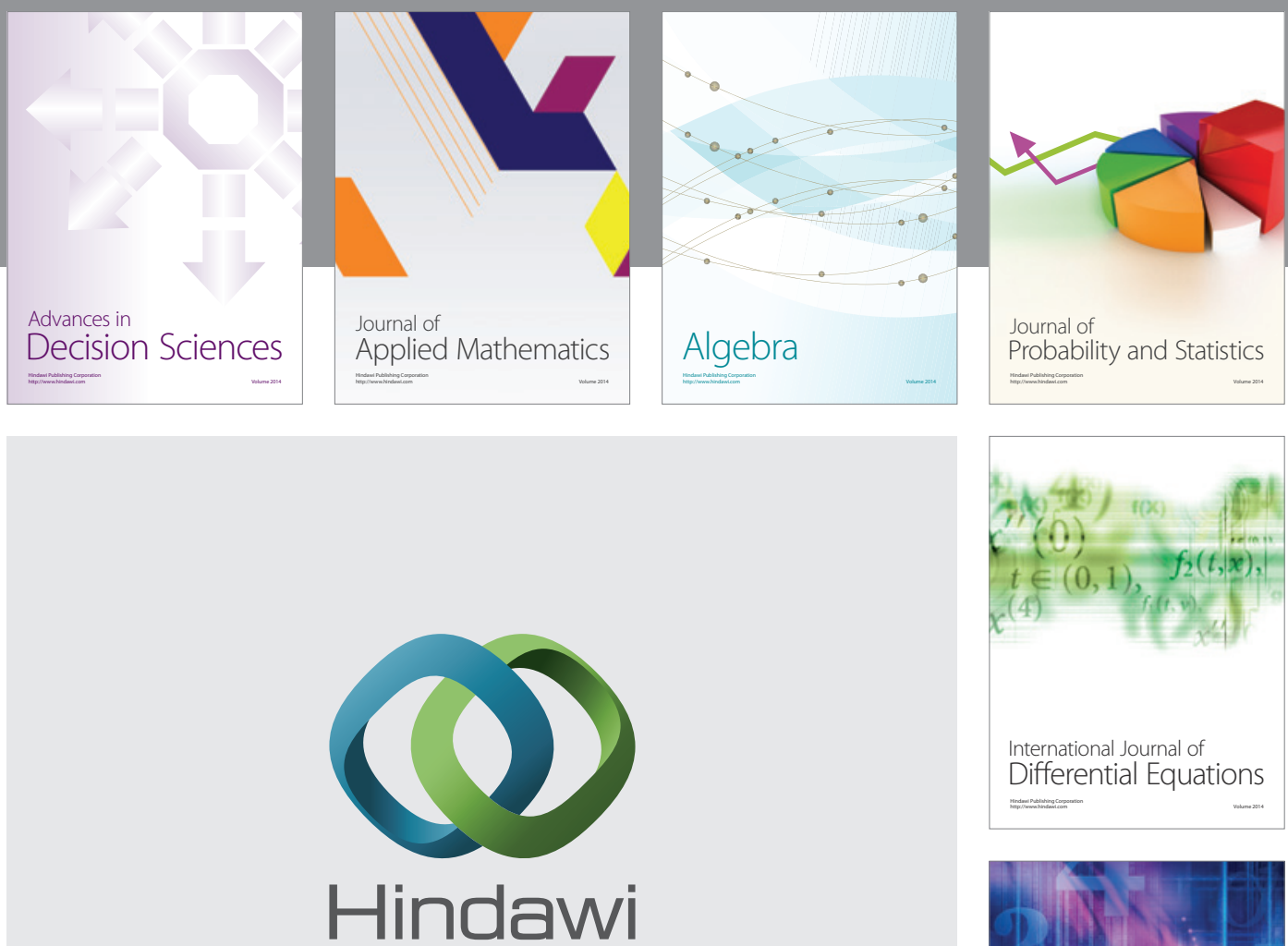

Submit your manuscripts at http://www.hindawi.com
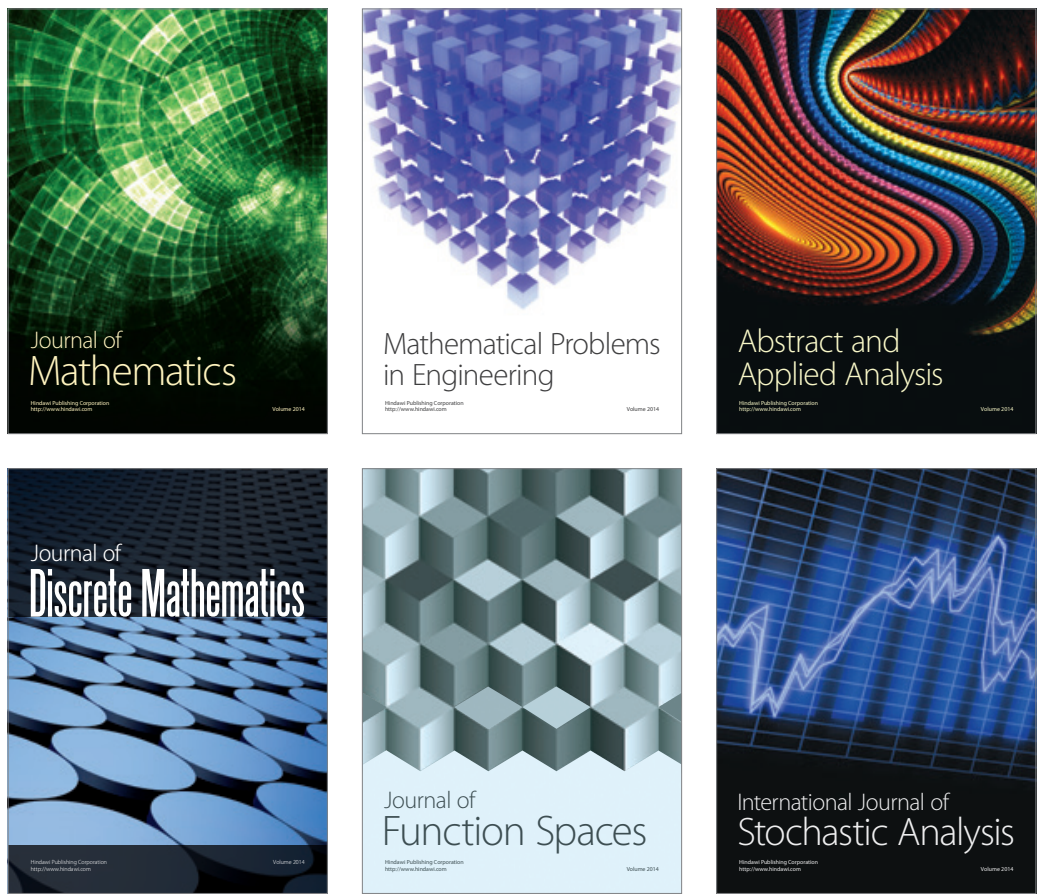

Journal of

Function Spaces

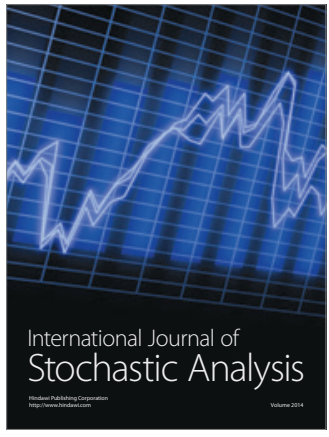

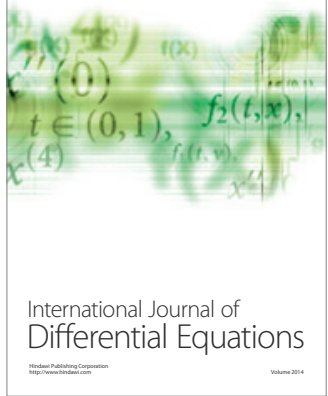
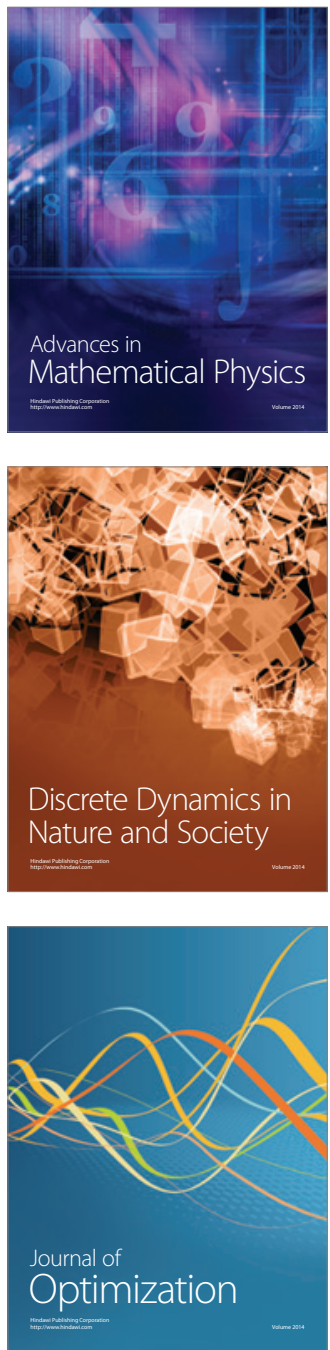